\title{
Somatic mutations in CTCF zinc fingers produce cellular phenotypes explained by structure- function relationships
}

Charles G Bailey ${ }^{1,2,3}$, Shailendra Gupta ${ }^{4,5}$, Cynthia Metierre ${ }^{1,2}$, Punkaja MS Amarasekera ${ }^{1,2}$, Patrick O’Young $^{1,2}$, Wunna Kyaw ${ }^{1,2}$, Tatyana Laletin ${ }^{1,2}$, Habib Francis ${ }^{1,2}$, Crystal Semaan $_{1,}{ }^{2}$, Krishna P Singh $^{4}$, Charles G Mullighan ${ }^{6}$, Olaf Wolkenhauer ${ }^{4,5,7}$, Ulf Schmitz ${ }^{2,3,8}$, John EJ Rasko ${ }^{2,3,9,10}$

${ }^{1}$ Cancer \& Gene Regulation Laboratory Centenary Institute, The University of Sydney, Camperdown, NSW, 2050, Australia

${ }^{2}$ Gene and Stem Cell Therapy Program Centenary Institute, The University of Sydney, Camperdown, NSW, 2050, Australia

${ }^{3}$ Faculty of Medicine \& Health, The University of Sydney, Sydney, NSW, 2006

${ }^{4}$ Department of Systems Biology and Bioinformatics, University of Rostock, 18051 Rostock, Germany

${ }^{5}$ Chhattisgarh Swami Vivekanand Technical University, Bhilai, Chhattisgarh 491107, India

${ }^{6}$ Department of Pathology and Hematological Malignancies Program, St. Jude Children's Research Hospital, Memphis, TN, 38105-3678, United States of America

${ }^{7}$ Stellenbosch Institute for Advanced Study (STIAS), Wallenberg Research Centre at Stellenbosch University, Stellenbosch, South Africa

${ }^{8}$ Computational Biomedicine Centenary Institute, The University of Sydney, Camperdown, NSW, 2050, Australia

${ }^{9}$ Cell and Molecular Therapies, Royal Prince Alfred Hospital, Camperdown, NSW, 2050, Australia ${ }^{10}$ Corresponding author 


\begin{abstract}
$\underline{\text { Background }}$

Human cancers commonly contain mutations in transcription factors that lead to aberrant DNA binding or altered effector function at target sites. One such factor significantly mutated in cancer is the evolutionarily-conserved CCCTC-binding factor (CTCF), which has fundamental roles in maintaining chromatin architecture and transcriptional regulation. Numerous cancer genome sequencing and functional studies have revealed CTCF's role as a haploinsufficient tumour suppressor gene. However, to date, structure-function relationships of somatic CTCF mutations have not been examined.
\end{abstract}

\title{
$\underline{\text { Methods }}$
}

We collated somatic CTCF mutations from cancer genome portals and published studies to determine their nature, frequency, distribution and potential functional impact. We undertook an in-depth examination of 5 CTCF missense zinc finger (ZF) mutations occurring within key intra- and inter-ZF residues. We performed functional analyses including cell growth, colony-formation, chromatin immunoprecipitation and transcriptional reporter assays. Based on their homology, each ZF mutation was then modelled on CTCF's ZF domain crystal structure and its structural impact analysed using molecular dynamics simulations.

\section{$\underline{\text { Results }}$}

We observed an enrichment of somatic missense mutations occurring in the ZF region of CTCF, compared to the unstructured $\mathrm{N}$ - and C-termini. Functional characterisation of CTCF ZF mutations revealed a complete (L309P, R339W, R377H) or intermediate (R339Q) abrogation as well as an enhancement (G420D) of the anti-proliferative effects of CTCF. DNA binding at select sites was disrupted and transcriptional regulatory activities abrogated. In silico mutagenesis revealed that L309P had the highest mutation energy and thus most severe impact on protein stability. Molecular 
docking and molecular dynamics simulations confirmed that mutations in residues specifically contacting DNA bases or backbone exhibited loss of DNA binding (R339Q, R339W, R377H). Remarkably, R339Q and G420D were stabilised by the formation of new primary DNA bonds. All mutations exhibited some loss or gain of bonds at neighbouring residues, often in adjacent zinc fingers.

\section{$\underline{\text { Conclusions }}$}

Our data confirm the significant negative impact haploinsufficient CTCF ZF mutations have on its tumour suppressor function. A spectrum of loss-, change- and gain-of-function impacts in CTCF zinc fingers are observed in cell growth regulation and gene regulatory activities. We have established that diverse cellular phenotypes in CTCF are explained by examining structure-function relationships.

\section{Keywords}

CTCF, tumour suppressor gene, haploinsufficient, somatic mutation, zinc finger, cancer, structural modelling, molecular docking, molecular dynamics simulation 


\section{Background}

Comprehensive catalogues of somatic mutations have been assembled from surveying the genomic landscape in numerous human cancers. More than 200 large-scale studies involving cancer types or subtypes of clinical or societal importance have been deposited in the cBio Cancer Genome Portal ${ }^{1}$. These studies have provided new insights into cancer causation and offered new leads for potential therapeutic intervention using a genomics-driven oncology approach. Cancers are remarkably heterogeneous in their distribution and frequency of somatic mutations. Paediatric cancers contain as few as 0.1 mutations per megabase $(\mathrm{Mb})$, whereas lung and melanoma samples may accumulate over $100 / \mathrm{Mb}$ (average 4.0/Mb) ${ }^{2}$. Whilst some genes are mutated at high frequencies, most genes are mutated at intermediate frequencies $(2-20 \%)^{3}$ adding to the complex molecular landscape underlying tumour biology. Those genes that exhibit mutation frequencies above background have been called significantly mutated genes, of which 127 have been identified amongst a dozen cancers ${ }^{4}$. These mutations disrupt diverse cellular processes including transcriptional regulation, histone modification, genome integrity, signalling and splicing ${ }^{4}$. Mut-driver genes (of which 138 have been identified) are a similar concept, whereby mutation or inactivation can cause a selective growth advantage in a direct or indirect manner ${ }^{5}$. Examples include inactivation of a tumour suppressor gene or activation of oncogenes.

In tumour cells, recurrent acquired mutations have been observed in nearly every DNA, RNA and protein component of normal transcriptional control ${ }^{6}$. These somatic mutations may directly impact transcription factors (TFs), their target sites, cis- and trans-regulatory elements as well as chromatin architecture leading to transcriptional dysregulation in cancer. Dysregulation of transcriptional programs in cancer cells can lead to transcriptional dependencies that offer opportunities for exploitation with targeted therapeutic strategies ${ }^{6}$. For example, pharmacological inhibition of the BET bromodomain-containing BRD4 protein has emerged as a promising therapeutic strategy to prevent MYC-dependent transcriptional signaling in various haemopoietic malignancies ${ }^{7-10}$. 
Investigating and exploiting these acquired cellular vulnerabilities is a major thrust of many cancer research efforts.

Approximately 1,600 to 2,000 TFs have been validated or predicted within the human genome ${ }^{11,12}$. TFs containing the zinc-coordinating $\mathrm{C} 2 \mathrm{H} 2$ class of DNA binding domains represent the largest class of transcription factors ${ }^{11}$, comprising nearly $50 \%$ of all $\mathrm{TFs}^{13}$. Human $\mathrm{C} 2 \mathrm{H} 2 \mathrm{TFs}$ contain an average of $\sim 10$ ZFs, specifying target sites of $\sim 30$ bases $^{14}$, however not all ZFs contact DNA simultaneously or indeed, are involved in DNA binding. Furthermore, the impact of somatic mutations on many TFs is unknown. Nor is it known whether such changes impact DNA binding or transcriptional activation globally or in a locus-specific manner.

One such $\mathrm{C} 2 \mathrm{H} 2-\mathrm{ZF}$-containing transcription factor, CCCTC-binding factor (CTCF), features a tandem array of 11 ZFs enabling multivalent binding to DNA target sites. Careful mutational analysis of key residues co-ordinating $\mathrm{Zn}^{2+}$ ion binding and $\mathrm{ZF}$ formation have shown key central $\mathrm{ZFs}$ that contribute binding to a core consensus site, whilst peripheral ZFs stabilise CTCF binding and bind additional conserved and non-conserved motifs ${ }^{15}$. Through combinatorial DNA binding and ZF multivalency, CTCF plays diverse roles in transcriptional regulation and three-dimensional genome organisation such that it has been called the 'master weaver' protein ${ }^{16}$. Unprecedented insights into nuclear organisation, obtained from high-resolution conformational maps of chromatin interactions, have defined the rules governing CTCF-mediated chromatin organisation. Firstly, CTCF links gene regulation to genomic architecture by co-ordinating DNA looping together with cohesin ${ }^{17-19}$. Secondly, CTCF defines the boundaries of topologically associating domains (TADs) ${ }^{20-22}$ in a structural framework that is evolutionarily conserved ${ }^{23}$. Depletion of CTCF can result in loss of DNA looping and insulation within TADs, however genomic compartmentalisation is preserved ${ }^{24}$. Lastly, TAD organisation is CTCF site orientation-specific ${ }^{23,25}$, such that rewiring or inverting CTCF sites 
can significantly perturb gene expression by affecting promoter-enhancer interactions or boundaries between euchromatin and heterochromatin ${ }^{26-28}$.

CTCF plays an integral role in cell-type specific genomic organisation and development. CTCF's role in development and differentiation has been examined in at least seven tissues or developmental stages in mice, zebrafish and Drosophila ${ }^{29}$. CTCF is absolutely essential, as CTCF null embryos are unable to implant ${ }^{30}$ and maintenance of CTCF expression ensures somatic cell viability ${ }^{31}$. Extensive characterisation of the action of CTCF in vitro and in vivo has led to its classification as a haploinsufficient tumour suppressor gene ${ }^{31-33}$. Whilst isolated somatic CTCF mutations were first identified in some solid tumours ${ }^{34}$, numerous cancer genome studies since have highlighted the impact and prevalence of CTCF mutation in multiple cancers ${ }^{4}$. CTCF is a significantly mutated gene in $\sim 20 \%$ of endometrial cancers s5-38 $^{38}$ and is recurrently mutated in myeloid and lymphoid malignancies ${ }^{39-42}$.

Despite many CTCF mutations having been identified in numerous cancer types, the functional consequences of these mutations have not been thoroughly examined. In this study, we performed a meta-analysis of all publicly available cancer mutation data for CTCF and showed a significant enrichment of missense mutations occurring in CTCF's ZF DNA binding domain. We have functionally characterised a subset of representative ZF mutations detected in acute lymphoblastic leukaemia samples to examine their consequences. Finally, we compared the impact of CTCF ZF mutation on DNA binding, transcriptional activation as well as on CTCF ZF domain structure using molecular modelling and molecular dynamics simulations. This is the first study to examine the effect of somatic mutation on CTCF ZF structure-function relationships. 


\section{Results}

\section{CTCF ZF domain is enriched for somatic missense mutations in cancer}

We analysed cancer genome sequencing databases and published mutation data to determine the distribution, frequency and nature of somatic mutations occurring in CTCF (Supplementary Table 1). The distribution and frequency of all known somatic mutations in CTCF is shown with recurrent mutant residues indicated (Figure 1A). The recurrent T204fs*26 and T204fs*18 mutations in CTCF arise due to a high frequency of insertions or deletions within a $30 \mathrm{bp}$ purine-rich $(>85 \%)$ region at c.1048 -c.1077 encoding T204. Frequently occurring missense or nonsense mutations occur at H284, S354, R377, R448 and R457 within the ZF region of CTCF (Figure 1A). Further analysis revealed that inactivating nonsense and frameshift mutations account for $\sim 40 \%$ of somatic CTCF mutations (Figure 1B). This result exceeds the '20/20 rule' for tumour suppressor gene classification which requires that $>20 \%$ of somatic mutations are inactivating ${ }^{5}$ and affirms our earlier work demonstrating CTCF's role as a tumour suppressor ${ }^{31-33}$. CTCF mutations occur prominently within hormoneresponsive cancers arising in the endometrium and breast ( 48\%) (Figure 1C).

We next examined somatic missense mutations and SNPs reported for CTCF and compared their observed and expected occurrences (Supplementary Table 2). CTCF's ZF domain has a significant enrichment for somatic missense mutations observed over the number expected for its relative size, such that the observed/expected $(\mathrm{O} / \mathrm{E})$ ratio $=1.47,(p<0.0001)$. Furthermore, there was a deenrichment of non-synonymous SNPs occurring within the ZF domain $(\mathrm{O} / \mathrm{E}=0.48, p<0.0001)$ (Figure 1D, Supplementary Table 2). These results suggest that the human CTCF ZF region is intolerant to normal genetic variation, but is frequently inactivated in cancer. As ZF mutations would likely affect DNA binding, these are likely to have a significant impact on CTCF function. There is a concomitant paucity of missense somatic mutations within the $\mathrm{N}$ - and $\mathrm{C}$-termini of $\mathrm{CTCF}(\mathrm{O} / \mathrm{E}=0.63, p<0.0001$ and $\mathrm{O} / \mathrm{E}=0.65, p=0.0269$ respectively, Figure 1D, Supplementary Table 2). Strikingly, the opposite trend is observed for SNPs in CTCF with an enrichment of missense SNPs in the N-terminus $(\mathrm{O} / \mathrm{E}=$ 
1.27, $p=0.0269)$ and C-terminus $(\mathrm{O} / \mathrm{E}=1.63, p=0.0032)$ Figure 1D, Supplementary Table 2). We then determined the potential functional impact of somatic missense mutations in CTCF using Polyphen analysis. Missense mutations exhibited an overall greater functional impact than missense SNPs $(0.80 \pm 0.35$ vs $0.49 \pm 0.44$, mean $\pm \mathrm{SD}, p<0.0001$, Supplementary Figure $1 \mathrm{~A})$. Further analysis indicated that there was a decrease in the ratio of transition to transversion mutations when comparing SNPs to missense somatic mutations (2.24 to 1.19 respectively, $p<0.0001$, Supplementary Figure 1B). These data provide further support for the role of CTCF as a tumour suppressor that is frequently mutated and functionally impacted in cancer.

As the majority of somatic missense mutations in CTCF occur within the ZF domain we next analysed the distribution of missense mutations in specific ZFs of CTCF. We found that the greatest proportion of mutations occurred in ZF4 ( 20\%), followed by ZF3 ( 15\%) (Figure 1E). ZFs 3-7 have been shown to be responsible for binding CTCF's core 15 bp consensus, with other ZFs providing binding specificity depending on adjacent motifs ${ }^{15,43}$. A sequence logo depicting all 11 ZFs in CTCF (10 $\mathrm{C} 2 \mathrm{H} 2-$ and $1 \mathrm{C} 2 \mathrm{HC}$-type) shows the conserved Cys and His residues that co-ordinate $\mathrm{Zn}^{2+}$ binding, an invariant hydrophobic Leu or Met residue at +4 and substantial amino acid variation at other positions (Figure 1F). The proportion of mutations occurring at each position within ZFs was determined. This analysis revealed that the proportion of inter-ZF mutations was $31.5 \%$, Cys/His mutations $(17.7 \%)$ and those affecting key DNA binding residues $(-1,+2,+3,+6,15.6 \%)$. Thus, approximately one-third of missense CTCF ZF mutations have an unknown impact but likely affect ZF folding and stability.

\section{CTCF ZF mutations exhibit loss- and gain-of-function in cell growth phenotypes in vitro}

To determine the functional consequences of CTCF ZF mutations, we examined missense mutations that had been detected in acute lymphoblastic leukaemia (ALL) samples: L309P (T-ALL; Mullighan unpublished) R339Q ${ }^{39}, \mathrm{R}^{3} 77 \mathrm{H}^{44}$ and G420D (diagnosis and relapsed hyperdiploid B-ALL; 
Mullighan unpublished) (Figure 2A, Supplementary Table 1). R377H occurs within the inter-ZF region, L309P affects the conserved intra-ZF Leu/Met residue, whilst G420D and R339Q both occur at key DNA contacting residues +2 and +6 , respectively (Figure $2 \mathrm{~A}$ ). We included $\mathrm{R} 339 \mathrm{~W}$ as a positive control as it was first identified in Wilms' tumour as a potential 'change-of-function' mutation that abrogated DNA binding to a subset of CTCF sites regulating genes involved in cell proliferation $^{34}$. All five mutations exhibit high Polyphen scores, indicating they significantly impact CTCF function (Figure 2A). We introduced these mutations into HA epitope-tagged human CTCF within a lentiviral expression vector that co-expresses eGFP via a $2 \mathrm{~A}$ peptide ${ }^{33}$. We transduced K562 erythroleukemia cells with CTCF WT and mutant constructs and showed that ectopic CTCF expression occurred at similar levels and above endogenous CTCF levels (Figure 2B). Immunofluorescent staining for ectopic HA-tagged CTCF indicated that all CTCF mutants maintained nuclear localisation similar to WT CTCF (Figure 2C). We next examined cell growth and showed that WT CTCF overexpression suppressed cellular proliferation $(p<0.0001)$ consistent with it being a tumour suppressor and as previously shown ${ }^{33}$ (Figure 2D). Mutants L309P, R377H and R339W abrogated the tumour suppressive effect of CTCF and exhibited cellular proliferation similar to the empty vector control (all $p<0.0001$ compared to WT), whilst R339Q had an intermediate effect on CTCF's anti-proliferative function ( $p<0.0001$ compared to WT; $p<0.001$ compared to control, Figure 2D). K562 cells expressing CTCF G420D exhibited similar proliferation to WT CTCF (Figure 2D). We next performed clonogenicity assays and showed that WT CTCF suppressed the colonyforming abilities of K562 cells as expected ( $p<0.0001$, Figure 2E). Again, L309P, R377H and R339W abrogated the suppressive effect of CTCF on colony formation $(p<0.0001)$ whilst R339Q had an intermediate effect compared to control $(p<0.0001)$ and a near-intermediate effect compared to WT ( $p=0.052$ ). Remarkably, G420D exhibited gain-of-function by further reducing the clonogenic capacity compared to WT (Figure 2E).

\section{CTCF ZF mutations disrupt transcriptional activity}


We next examined the impact of ZF mutations on transcriptional regulation by CTCF. Frequently occurring $\mathrm{N}$-and C-terminal somatic missense mutations (Y226C and R603C respectively) were included as additional controls. Y226 is a key anchoring residue in the interaction of CTCF with the SA2-SCC1 cohesin complex ${ }^{45}$, whilst R603 resides within the nuclear localisation signal. Lentiviral plasmids encoding WT or mutant CTCF (Figure 3A) were transfected into HEK293T cells followed by quantitation of CTCF protein and eGFP fluorescence levels. All CTCF ZF mutants exhibited decreased levels of ectopic CTCF expression to levels $\sim 10-20 \%$ of WT, whilst non-ZF mutants demonstrated levels comparable to, or higher than, WT control (Figure 3B \& C). WT CTCF suppressed eGFP expression compared to eGFP control $(p<0.0001$, Figure 3C) whilst decreased eGFP expression was observed with CTCF mutants R339Q, R339W, R377H and G420D compared to WT. L309P had no impact; however, non-ZF mutations (Y226C and R603C) exhibited higher eGFP expression than WT ( $p=0.0248$ and $p=0.0061$ respectively), but similar to empty vector control (Figure 3D). These data suggested that ectopic plasmid-encoded CTCF could regulate its own expression. This was supported by the prediction of over a dozen putative CTCF binding sites in the CTCF WT and mutant plasmid backbone including within the CMV promoter that drives viral RNA expression (horizontal dashes, Figure 3A). Accordingly, CTCF ZF mutants exhibited diminished CTCF protein expression and lower eGFP expression (Figure 3B, C \& D). Collectively, these data indicate that CTCF ZF mutants impact on CTCF's normal role as a transcriptional regulator, which most likely results from disruption or destabilisation of DNA binding.

To examine this, we performed chromatin immunoprecipitation (ChIP) to determine if ZF-mutant disruption of transcriptional regulation leads to abrogation or alteration of DNA binding at CTCF target sites. Notably, we achieved equivalent levels of HA-tagged WT and ZF mutant CTCF in K562 cells after lentiviral transduction $(\sim 15-20 \%$ for all, Supplementary Figure 2$)$. We then performed ChIP using an anti-HA antibody, followed by PCR amplification of known CTCF target sites (Figure 4). We observed both WT and mutant CTCFs still associating with archetypal CTCF target sites such 
as the H19 imprinting control region (ICR) and the $\beta$-globin hypersensitivity site HS5 (Figure 4A). However, variegated CTCF mutant binding was detected at other cognate CTCF target sites proximal to the regulatory regions of $B A G 1, M A G E A 1, X I S T, B R C A 1, P L K$ and $A P P \beta$ (Figure 4A). All CTCF ZF mutants exhibited a selective loss of DNA binding, with L309P, R339Q and R337H mutations exhibiting the greatest loss in binding (Figure 4A-E). All CTCF mutants except G420D exhibited some loss of binding within the archetypal CTCF-regulated gene $C$-MYC (Figure 4B). CTCF binding sites within known enhancers (Figure 4C), insulator sites (Figure 4D) and TAD boundaries (Figure 4E) all showed selective binding by most CTCF ZF mutants. As CTCF binding is not completely abrogated, these data are consistent with CTCF ZF mutants displaying a change-of-function rather than loss-of-function.

\section{Molecular dynamics (MD) simulations explain CTCF loss- and gain-of-function ZF mutant phenotypes}

To gain insights into the structural impact of these mutations we modelled them on the published crystal structure of CTCF's ZF domain (ZFs 2-7) in complex with DNA ${ }^{43}$ and performed molecular dynamics (MD) simulations. The locations of the 4 mutated ZF residues were superimposed on the CTCF structure (Figure 5A). The folding free energy change $(\Delta \Delta \mathrm{G})$ calculated for all 5 resulting ZF mutations indicate that each of the mutations are destabilizing. L309P is predicted to have the most severe impact on CTCF folding $(\Delta \Delta \mathrm{G}=12.05 \mathrm{kcal} / \mathrm{mol})$, compared to R339Q $(\Delta \Delta \mathrm{G}=6.87 \mathrm{kcal} / \mathrm{mol})$, R339W $(\Delta \Delta \mathrm{G}=5.00 \mathrm{kcal} / \mathrm{mol}), \mathrm{R} 377 \mathrm{H}(\Delta \Delta \mathrm{G}=5.64 \mathrm{kcal} / \mathrm{mol})$ and $\mathrm{G} 420 \mathrm{D}(\Delta \Delta \mathrm{G}=1.91 \mathrm{kcal} / \mathrm{mol})$ (Figure 5B). Time evolution studies of secondary structure in WT and mutant CTCF ZF domain indicate that structural elements are stable at the location of each mutation (Supplementary Figure 3). However, $\beta$-sheet-forming elements (red) are disrupted by: L309P (ZF2), R339Q, R339W (ZF3) and R377H (ZF4-5) between aa 353 - 363 in ZF4; and R339W, R377H and G420D (ZF6) between 295305 in ZF2. In all mutants, the $\beta$-sheet and turn structure at aa $408-418$ (ZF6) is also disrupted (Supplementary Figure 3). 
To examine each mutation in more detail, we examined the superimposed structures of WT and mutant CTCF ZF structures. L309 is facing away from DNA and does not directly contact DNA either before or after mutation to Pro (Figure 5C). Despite this, analysis of molecular interactions between neighbouring CTCF amino acid residues and DNA revealed 7 existing bonds were lost, whereas 12 new bonds were formed (Supplementary Table 3). Root-mean-square deviation (RMSD) measurements showed that the L309P mutation induced a substantial increase in the deviation of the ZF2 backbone compared to WT over the 10 ns simulation run (Figures 6A \& B, $p<0.0001$ ). Similarly, root-mean-square fluctuation (RMSF) measurements spanning the entire ZF 2-7 structure (Supplementary Figure 4) indicated that there was a considerable increase in flexibility $(p<0.0001$, Figure 6C). Consequent to all the conformational changes, the distance of the ZF2 centroid from the DNA centroid was also increased $(0.916 \AA)$ in the L309P mutation (Figure 6D).

Arginine 339 at DNA-binding position ' +6 ' within ZF3 directly contacted guanine (G14) and cytosine (C13) residues on one DNA strand via two hydrogen bonds and one cation- $\pi$ bond, however mutation to Q (R339Q) or W (R339W) abolished these bonds (Figure 5D \& E, Supplementary Table 3). Remarkably, Q339 formed two new hydrogen bonds: firstly, between the Gln side chain carbonyl group and cytosine (C15); and secondly, between the side chain amide group and thymine (T7) on the complementary strand (Figure 5D). Both mutations also disrupted the interaction of E336 with cytosine (C15), with 6 and 4 new bonds formed at neighbouring residues for Q339 and W339 respectively (Supplementary Table 3). MD simulations showed that the R339Q triggers less conformational deviation than WT or R339W (Figures 6A \& B, both $p<0.0001$ ), however over the entire ZF structure R339Q and R339W both exhibited more flexibility than WT (Figure 6C, $p=$ $0.0018 \& p<0.0001$ respectively). Consequently, R339Q shifted ZF3 towards DNA (2.342 $\AA$ ) and in the case of R339W, ZF3 moved away from DNA (3.021 $\AA$ ) (Figure 6D). 
$\mathrm{R} 377 \mathrm{H}$, which occurs in an inter-ZF residue between ZF4 and ZF5, disrupted three hydrogen bonds that stabilise the interaction of R377 with the DNA phosphate moiety at guanine (G8) (Figure 5F). Adding to this, 22 neighbouring molecular contacts are lost and 22 new bonds are formed (Supplementary Table 3). RMSD measurements show that R377H induced an increased deviation in the conformation over time (Figures $6 \mathrm{~A} \& \mathrm{~B}, p=0.0439$ ) and increased flexibility in the entire ZF structure (Figure 6C, $p<0.0001$ ). As a result, both ZF4 and ZF5 were shifted away from the DNA phosphate backbone $(1.643 \AA \& 2.718 \AA$ respectively, Figure $6 \mathrm{D})$.

Finally, CTCF modelling confirms glycine at position 420 and DNA-binding position ' +2 ' in ZF6 does not directly contact DNA (Figure 5G). However, when mutated to aspartic acid (G420D), a new hydrogen bond is formed between the side chain carbonyl group and cytosine (C16) in the core consensus sequence (Figure 5G). A net loss of 4 bonds at neighbouring residues was also observed (Supplementary Table 3). RMSD measurements showed that G420D exhibited decreased structural deviation during the simulation run (Figures $6 \mathrm{~A} \& \mathrm{~B}, p<0.0001$ ) and decreased RMSF values compared to WT indicating reduced flexibility $(p<0.0001$, Figure 6C). Consequently, G420D resulted in ZF6 shifting $1.841 \AA$ toward the DNA (Figures 6D).

In summary, our data suggest that mutations R339W and R377H disrupted CTCF's primary interactions with DNA and, along with the highly destabilising L309P mutation, are responsible for shifting ZF domains away from DNA. Importantly, R339Q and G420D both formed new primary bonds and the associated ZF domain moved nearer to the DNA. 


\section{Discussion}

Tumour-specific mutations in CTCF were described almost two decades ago ${ }^{34}$. In that seminal report, functional testing of some ZF mutations comprised electrophoretic mobility shift assays (EMSA) and a reporter assay. The key finding from these preliminary functional studies was that these somatic CTCF ZF mutations exhibited selective disruption in DNA binding to some, but not all, CTCF target sites, giving rise to the concept of 'change-of-function' mutations ${ }^{34}$. Since then the functional characterisation of CTCF ZF mutations has been limited, despite many landmark cancer genome studies reporting hundreds of missense somatic CTCF ZF mutations.

The potential genome-wide impacts of CTCF ZF mutation on DNA binding were examined through mutation in all $11 \mathrm{ZFs}$ of a key $\mathrm{Zn}^{2+}$ co-ordinating histidine residue within the conserved $\mathrm{C} 2 \mathrm{H} 2$ tetrahedron arrangement that co-ordinates ion binding ${ }^{15}$. This approach, whilst not directly emulating tumor-specific missense mutations, revealed that in all cases DNA binding was not completely abolished. Indeed, residual DNA binding ranged from $\sim 15-80 \%$ depending on the position of the $\mathrm{ZF}^{15}$. Our previous report showed that the 3 CTCF ZF mutations most frequently occurring in endometrial cancers (K365T, R377H, P378L) had differing impacts on CTCF function when overexpressed ${ }^{38}$. The two inter-ZF mutations (R377H and P378L) abrogated CTCF's anti-proliferative and anticlonogenic effect in Ishikawa endometrial cancer cells. However, mutation of K365, a key DNAbinding residue at position ' +3 ' in $\mathrm{ZF} 4$, to threonine, had no impact on proliferation or colony formation $^{38}$, despite causing a 20 -fold loss of CTCF DNA-binding affinity ${ }^{43}$. Interestingly, CTCF K365T conferred significantly increased resistance to UV-induced apoptosis in Ishikawa cells compared to WT CTCF, suggesting the first pro-tumourigenic role of CTCF ZF mutations ${ }^{43}$.

Initial deletion mutagenesis studies of the CTCF ZF domain indicated ZFs 3-11 were required to bind the human $c$-myc promoter ${ }^{46}$. Further refinement was achieved in two studies, in which similar binding modes were confirmed on the chicken $c$-myc promoter requiring central ZFs $2-7^{46}$ or ZFs 3- 
$8^{47}$. Furthermore, the central ZFs 5-7 of CTCF were required for $A P P \beta$ promoter binding, however it was the peripheral ZFs which provided the stability in DNA binding ${ }^{47}$. Whilst gel mobility shift analysis confirmed that CTCF ZFs 4-7 were necessary and sufficient to bind to a core 12 bp consensus sequence $^{48}$, the first crystal structure of the CTCF ZF domain resolved that ZFs 3-7 bound the 15 bp core DNA consensus sequence ${ }^{43}$. Nakahashi et al proposed a 'saddle' model containing a core CTCF motif (C) bound by central ZFs 4-7 as well as upstream (U) and downstream (D) modules bound by peripheral $\mathrm{ZFs}^{15}$. Yin et al further refined this model by describing 4 CTCF binding site modules ${ }^{49}$. Modules 4, 3 and 2, spanning the 15 bp core CTCF consensus as well as downstream sequences, are bound by ZF3, ZFs 4-6 and ZF7 respectively; whilst upstream module 1 is bound by ZFs 9-1149. These studies provide insights as to why mutations occurring in different CTCF ZFs may produce diverse effects on DNA binding and functional outcomes depending on where in the modular binding mode the mutant $\mathrm{ZF}$ residue occurs.

The five different somatic missense ZF mutations we examined in this study each occur in key positions within the central zinc fingers. Each mutation provided critical insights into CTCF structurefunction relationships. The spatial arrangement of residues within the $\mathrm{C} 2 \mathrm{H} 2 \mathrm{ZF}$ finger motif, including the flexible inter-ZF link, are critical to maintaining ZF structure, and are therefore very highly conserved ${ }^{50}$. Somatic ZF mutations did not affect overall CTCF protein stability or localisation within the nucleus when stably expressed. However, when transiently overexpressed, CTCF ZF mutants clearly decreased transcriptional activation compared to WT CTCF. Interestingly, our previous endometrial cancer study indicated that missense ZF-containing CTCF alleles were expressed at a higher frequency than WT alleles, when comparing RNA sequencing to DNA sequencing ${ }^{38}$. This suggested that expression of aberrant CTCF was up-regulated to functionally compensate for a deficit in CTCF function. In our study, we observed differing impacts on CTCFmediated cellular proliferation. These impacts on CTCF function are attributable to a change or gain in DNA-binding specificity. 
L309 typifies an invariant hydrophobic residue (always Leu or Met) in the alpha helical region of all CTCF C2H2 ZF fingers. Residues near the C-terminal end of each C2H2 ZF fold into an alpha helix, positioning key amino acids within the helix to interact directly with DNA ${ }^{50}$. L309 mutation to Pro (L309P) affected the thermodynamic stability of ZF2, most likely through the $\alpha$-helical-breaking tendency of proline in water-soluble proteins. We confirmed increased RMSD and RMSF values during molecular dynamics simulations and a shift of CTCF away from DNA. Not surprisingly, despite some DNA-binding still being maintained, L309P exhibited loss-of-function in in vitro cell growth assays. R339 mutation to Q or W differentially impacted CTCF growth and colony forming function and this too was explained by molecular dynamics simulations. For R339Q, the ZF domain of the CTCF shifts closer to DNA and two new hydrogen bonds are formed at Q339, explaining the intermediate loss-of-function cell growth phenotype observed with R339Q. R339W, which exhibits loss-of-function phenotypes, disrupts all primary DNA contacts and deflects ZF3 away from DNA. This is despite R339W still maintaining nearly half (47\%) of DNA binding genome-wide ${ }^{15}$.

R377 resides in one of the inter-ZF regions which act as bridges between ZFs to allow flexibility in the DNA-free form but stability in the DNA-bound form ${ }^{43}$. Our modelling showing that R377 contacts the DNA phosphate backbone was also confirmed by structural studies of the CTCF ZF domain bound to the $P c d h$ enhancer ${ }^{49}$. Hence, not all amino acids at canonical DNA binding positions in ZFs directly contact DNA, such that intra- and inter-ZF residues are also involved in DNA contacts $^{49}$. The $\mathrm{R} 377 \mathrm{H}$ mutation, which eliminates this DNA interaction, also destabilises neighbouring molecular interactions. R377H exhibited loss-of-function cell growth phenotypes in K562 erythroleukaemia cells, similar to our observations in endometrial cancer cells ${ }^{38}$. Remarkably, despite G420D exhibiting some loss of binding to target sites and loss of gene regulatory activity, a gain-of-function was observed as it suppressed colony formation to a greater extent than WT CTCF. Consistent with these phenotypes, G420D formed a new bond with DNA and resulted in ZF6 shifting 
towards DNA. Overall, these studies reveal that the location of the ZF missense mutation determines the impact it has on loss-, change- or gain-of-function. We examined mutations in DNA-contacting residues, in a residue co-ordinating ZF folding, in the inter-ZF region and within or outside the central core consensus binding ZFs 4-7. Furthermore, the mutant amino acid residue side chain can also have a significant impact on cellular phenotypes.

Different ZF modules with identical DNA specificity residues (at positions $-1,+2,+3$ and +6 ) can bind different sequences, influenced by DNA sequence context and inter-ZF residue-residue interactions $^{51}$. Furthermore, neighbouring ZFs may affect the DNA-binding conformation and specificity of a particular $\mathrm{ZF}^{52}$. Our data has revealed that those residues in close proximity to the mutant residue can lose existing bonds or acquire new DNA interactions. Missense ZF mutations in CTCF can destabilise the DNA-bound conformation of neighbouring ZFs. Thus, MD simulations have illuminated the broad and diverse impact that CTCF ZF mutations exert on DNA binding.

Human $\mathrm{C} 2 \mathrm{H} 2$ ZF-containing TFs contain on average $10 \mathrm{C} 2 \mathrm{H} 2-\mathrm{ZF}$ domains, leading to possible binding sites of up to $\sim 30 \mathrm{bp}$. These suffice to specify target sites in the human genome $\mathrm{H}^{14}$. However, not all CTCF ZFs bind DNA simultaneously, meaning some may be oriented for RNA or proteinprotein interactions. Thus, the impact of somatic CTCF ZF missense mutations on: i) RNA interactions; ii) protein-protein interactions; and iii) higher-order chromatin structure has yet to be examined. CTCF is also a high affinity RNA-binding protein conferring long-range chromatin interactions mediated via $\mathrm{RNA}^{53}$. Jpx, a long non-coding RNA known to activate Xist, interacts with CTCF and relieves CTCF-mediated repression at the Xist locus, suggesting that Jpx directly interferes with CTCF DNA-binding via its ZF domain ${ }^{54}$. In CTCF, ZFs 10 and 11 in concert with the C-terminus have been defined as an RNA-binding region, binding the long noncoding RNA Wrap $53^{55}$. Mutations in peripheral ZFs 1 and 10 can disrupt CTCF interactions with RNA, leading to disruption of CTCFmediated chromatin looping and higher-order chromatin structure ${ }^{55}$. 
Most $\mathrm{C} 2 \mathrm{H} 2$-containing proteins interact with a diverse range of proteins, defining their role as transcriptional activators or repressors ${ }^{13}$. This confers additional gene regulatory complexity at specific DNA sites, potentiating the disruption of locus-specific gene expression as a result of ZF mutations. CTCF homodimerisation, which is essential for correctly orientating CTCF DNA looping and topology, is orchestrated via inter-CTCF interactions between the ZF domain and C-terminus ${ }^{56}$. The CTCF ZF domain interacts with TFs YB1, OCT and SIN3A as well as the chromatin remodeler $\mathrm{CHD}^{57}$, amongst other proteins. The proteome-wide impact of somatic CTCF ZF missense mutations on homodimerisation and protein-protein interactions is yet to be elucidated.

Finally, it is not known what impact somatic ZF missense mutations will have on rewiring genomic architecture. Topologically associated domains (TADs) are discrete territories, compartmentalising the genome into independent, often evolutionarily conserved domains $20,21,23,58,59$. TADs are characterized by frequent CTCF-mediated contacts within domains and a low frequency of contacts between domains ${ }^{60}$. These TADs are themselves demarcated into sub-megabase sub-TADs and loop domains, often differentially co-ordinated by CTCF interaction with other architectural proteins such as cohesin ${ }^{19,24,61}$. Deletion or inversion of CTCF sites at TAD boundaries can drastically affect gene regulation, leading to ectopic activation of gene expression due to illegitimate promoter and enhancer interactions, often with pathogenic consequences $26,27,62,63$. In cancer, genetic alteration or hypermethylation of CTCF sites at TAD boundaries can disrupt chromatin topology and lead to aberrant activation of oncogenes ${ }^{64-66}$. The global impact of somatic missense mutations in CTCF, which typically only occur on one allele and cause CTCF haploinsufficiency, remains to be investigated.

\section{Conclusions}

Over the last decade, unprecedented insights into CTCF's essential role in genome organisation and architecture have been revealed via the generation of high-resolution maps of chromatin interactions 
by chromosome conformation capture (3C)-based techniques. However, the structure-function relationships of CTCF mutations, particularly within the DNA-binding ZF domain, have not been investigated. We reveal that the CTCF ZF domain is significantly mutated in cancer, with ZF-specific missense mutations impacting CTCF's anti-proliferative capacity, DNA-binding and gene regulatory activities. Strikingly, we observed a broad spectrum of functional impacts ranging from complete, partial or no loss-of-function in cellular growth phenotypes and transcriptional regulation, as well as gain-of-function, resulting from the formation of new bonds between the mutant ZF and DNA. Our MD simulations revealed that all CTCF ZF mutations were destabilising, with the loss or gain in DNA binding not just localised to the mutant residue. This highlights the importance of understanding structure-function relationships in normal and mutated CTCF. As CTCF exhibits haploinsufficiency in cancer, the interplay between mutant and wildtype CTCF at specific loci and at target sites genomewide remains an unanswered question. Understanding how somatic CTCF ZF mutations affect RNA, protein-protein or chromatin interactions globally will be the next frontier in understanding the molecular pathophysiology of cancer. 


\section{Materials and Methods}

\section{Cell lines}

Human erythroid leukaemia (K562) cells were grown in RPM1 1640 medium and human embryonic kidney (HEK293T) cells were cultured in DMEM. All basal media were supplemented with 10\% FCS $(\mathrm{v} / \mathrm{v})$, penicillin $(100 \mathrm{U} / \mathrm{mL})$ and streptomycin $(100 \mu \mathrm{g} / \mathrm{mL})$. All human cell lines were previously authenticated by short tandem repeat profiling (Cellbank, Australia).

\section{Reagents: Expression Vectors and Antibodies}

The lentiviral vector pCCLteteGFP2AHAhCTCF ${ }^{33}$ was used to express CTCF ZF mutations. PCR amplicons containing ZF mutations (L309P, R377H, G420D) were generated by splice overlap extension PCR and were cloned in using BmgBI/ClaI. R339Q and R339W mutations were created by gene synthesis from DNA2.0 and sub-cloned using Bsu36I/Tth111I. Y226C and R603C were synthesized as Geneblocks (IDT) and cloned into BstXI/BstXI sites and PstI/BlpI sites respectively. Primary antibodies include: CTCF rabbit monoclonal (\#3418, Cell Signaling Technology; 1:5,000), HA epitope mouse monoclonal (HA.11, Covance; 1:5,000) and GAPDH (ab8245, Abcam; 1:5,000). Secondary antibodies include: rabbit or mouse antibodies conjugated to horseradish peroxidase (HRP, Millipore; 1:5,000).

\section{Retroviral and lentiviral transduction}

Viral supernatants were produced by calcium phosphate transfection of HEK293T cells: with pJK3, pCMVTat and pL-VSV-G packaging plasmids used to produce replication-defective retroviruses; and pRSV-Rev, pMDLg/p.rre and pMD2.VSV-G used to produce replication-defective lentiviruses. Viral supernatants collected after $24-48 \mathrm{~h}$ were $0.45 \mu \mathrm{M}$-filtered and snap-frozen or concentrated by ultracentrifugation for $2 \mathrm{~h}$ at 20,000 rpm in a SW28 Beckman rotor. Viral supernatant was resuspended on ice in $10 \%(\mathrm{v} / \mathrm{v}) \mathrm{FCS} / \mathrm{DMEM}$ at 1/100th of the original volume. Attached cells (1- 
$5 \times 10^{5} /$ well) were seeded in 6-well plates before addition of fresh medium containing viral supernatant $\left(\sim 5 \times 10^{5}\right.$ transducing units) and Polybrene ( $8 \mu \mathrm{g} / \mathrm{mL}$; Sigma) and 'spin-oculated' for 90 min at 1,500 rpm. The supernatant was replaced with medium $12 \mathrm{~h}$ post-transduction and fluorescent cells were purified $24 \mathrm{~h}$ later by fluorescence-activated cell sorting (FACS; $>95 \%$ purity on re-analysis) using a FACS Influx (Becton Dickinson, BD). K562 cells $\left(\sim 5 \times 10^{5} / \mathrm{mL}\right)$ in $1 \mathrm{~mL}$ medium with $4 \mu \mathrm{g} / \mathrm{mL}$ Polybrene were placed in a $5 \mathrm{~mL}$ capped FACS tube and transduced with viral supernatant for $90 \mathrm{~min}$ by 'spin-oculation'. The cells were resuspended, incubated at $37^{\circ} \mathrm{C}$ for $4 \mathrm{~h}$ before removal of viral supernatant. For in vitro assays, cells were either plated out immediately or allowed to recover after sorting for $48-72 \mathrm{~h}$ in medium containing $100 \mu \mathrm{g} / \mathrm{mL}$ Normocin (Invivogen).

\section{Immunofluorescence}

Transduced K562 cells $\left(1 \times 10^{6}\right)$ were fixed with equal volume of 4\% (w/v) formaldehyde for $20 \mathrm{~min}$ at room temperature (RT). Cells were centrifuged at 2,000 rpm for $3 \mathrm{~min}$ and resuspended in PBS twice. Cells $\left(5.0 \times 10^{5}\right)$ were allowed to settle onto coverslips coated with poly-D-lysine, before drying and permeabilisation with Triton X-100 0.5\% (v/v) in PBS for $10 \mathrm{~min}$ at RT. Cells were rinsed three times in PBS and blocked in 3\% (w/v) BSA/PBS for 40 min at RT. Cells were rinsed three times in PBS and incubated with mouse anti-HA antibody (1:500, HA.11, Covance) for 90 min at RT. Cells were rinsed three times in PBS and incubated with F(Ab')2-goat anti-mouse IgG-Alexa 594 (1:500, \#A11020, ThermoFisher Scientific) and DAPI (1:10,000, \#D1306, Life Technologies) for 40 min at RT. Cells were rinsed three times in PBS and mounted using Prolong Gold Antifade (Life Technologies). Slides were imaged at 60x using the DeltaVision Personal (Applied Precision) and the DAPI, FITC and A594 filters. Images were analysed after deconvolution using Volocity software.

\section{Western blot analysis}

Protein extracts were prepared with cell lysis buffer containing $20 \mathrm{mM}$ TrisCl (pH 8), $\mathrm{NaCl}(150$ $\mathrm{mM}), 1 \%(\mathrm{v} / \mathrm{v})$ Triton X-100, 0.1\% (v/v) SDS, 0.5\% (w/v) sodium deoxycholate and EDTA-free 
protease inhibitor cocktail (cOmplete, Roche Life Science), prior to separation using denaturing sodium dodecyl sulfate polyacrylamide gel electrophoresis (SDS-PAGE). Proteins were transferred in a semi-dry transfer apparatus to PVDF membrane before immunoblotting. Membranes were blocked in PBST containing 20\% (v/v) BlokHen (AvesLab) or PBST containing $0.3 \%(\mathrm{w} / \mathrm{v})$ BSA, 1\% (w/v) polyvinylpyrrolidone, $1 \%$ PEG (mw 3350). Protein expression was detected using primary antibodies followed by washing and staining with appropriate secondary antibodies against rabbit, goat or mouse IgG conjugated to horseradish peroxidase (HRP). The HRP substrate SuperSignal $®$ Chemiluminescent Substrate (Pierce) was detected on a Kodak Imagestation 4000R Pro or BioRad Chemidoc Touch. Blots were stripped with ReBlot Plus (Merck Millipore) prior to re-probing with protein loading control antibodies.

\section{Mutation and Bioinformatic analysis}

CTCF mutations were obtained from the Catalogue of Somatic Mutations in Cancer (COSMIC) portal, The Cancer Genome Atlas (TCGA) cBIO portal and published reports (see Supplementary Table 1). Single nucleotide variants were obtained from dbSNP. The potential impact of mutations was determined using Polyphen-2. All amino acid alignments were performed using the Clustalw algorithm within MacVector. A raw alignment of CTCF ZFs was exported into Weblogo (weblogo.berkeley.edu/logo.cgi) to generate a sequence logo. The maximum sequence conservation for an amino acid is $\log _{2} 20 \sim 4.32$ bits. CTCF target sites in CTCF-expressing plasmid pCCLteteGFP2AHA-hCTCF were predicted using MatInspector (Genomatix).

\section{Cell biological assays}

Cell proliferation was assessed by 3-(4,5-1,2 methylthiazol-2-yl)-2,5-diphenyltetrazolium bromide (MTT) assay (Merck Millipore). K562 cells (5,000/well) were plated in triplicate in a 96-well plate and proliferation was assessed over $4 \mathrm{~d}$ by the addition of MTT at $37^{\circ} \mathrm{C}$ overnight. The reaction was quenched with isopropanol/ $\mathrm{HCl}$ and then absorbance was measured at $572 \mathrm{~nm}$ using a Wallac 1420 
Victor plate reader (Perkin Elmer). The clonogenic capacity of K562 cells was measured by plating 5,000 cells diluted in Iscove's Modified Dulbecco Medium (Life Technologies) containing $3 \mathrm{~mL}$ Methocult GF H4230 (Stem Cell Technologies) and plated in triplicate in $35 \mathrm{~mm}$ gridded plates and incubating for 8-10 d.

\section{Chromatin Immunoprecipitation}

K562 cells $\left(1 \times 10^{6}\right.$ in $\left.1 \mathrm{~mL}\right)$ were transduced with $10-60 \mathrm{uL}$ viral supernatant of the control (eGFP only), CTCF WT and five CTCF mutants. After $72 \mathrm{~h}$ the cells were assessed by flow cytometry (LSR Fortessa, Becton Dickinson) and shown to vary between $\sim 14-21 \%$ expression. For each chromatin immunoprecipitation (ChIP), $5 \times 10^{6}$ cells were cross-linked with $1 \%(\mathrm{w} / \mathrm{v})$ formaldehyde for $10 \mathrm{~min}$ and then quenched with $1 \mathrm{M}$ glycine to a final concentration of $20 \mathrm{mM}$. Nuclear lysates were sonicated for 25 cycles, $30 \mathrm{~s}$ on, $30 \mathrm{~s}$ off using a Bioruptor sonicator (Diagenode). For each immunoprecipitation, $3 \mu \mathrm{g}$ of rabbit polyclonal antibody against the HA epitope (ab9110, Abcam) was used. Magna ChIP тм Protein A/G conjugated magnetic beads (Millipore) were used to immunoprecipitate antibody-bound chromatin complexes, and all subsequent steps were performed according to the manufacturers' instructions. After de-crosslinking, phenol/chloroform extraction, and ethanol precipitation, PCR was performed on genomic DNA targets using Phusion polymerase with GC buffer (Finnzyme). PCR primers spanning experimentally validated CTCF targets sites were designed from previous reports (see Supplementary Table 4). A more detailed protocol is available on request.

\section{Transfection of WT and mutant CTCF}

HEK293T cells $\left(1 \times 10^{5}\right)$ were plated into 12 -well plates, $18 \mathrm{~h}$ before transfection. In each transfection, pCCLteteGFP2AHACTCF WT, mutant or empty vector $(0.5 \mu \mathrm{g})$ was combined with $2 \mu \mathrm{L}$ Lipofectamine 2000 (ThermoFisher) in OptiMEM medium (Gibco) according to the manufacturers' 
bioRxiv preprint doi: https://doi.org/10.1101/2021.01.08.425848; this version posted January 8,2021 . The copyright holder for this preprint (which was not certified by peer review) is the author/funder, who has granted bioRxiv a license to display the preprint in perpetuity. It is made available under aCC-BY-NC-ND 4.0 International license.

instructions. After $48 \mathrm{~h}$, cells were detached and assessed for eGFP by flow cytometer (LSR Fortessa, Becton Dickinson) and then lysed with cell lysis buffer.

\section{Structural Modelling and MD simulations}

A $3.2 \AA$ X-ray diffraction crystal structure representing the CTCF ZFs 2-7 / DNA complex (PDB: $5 \mathrm{TOU})^{43}$ was used as the initial template to prepare CTCF mutant models. The template was optimized using 'Prepare Protein' and 'Energy Minimization' protocols available in Biovia Discovery Studio (DS) 2017R2 software suite. Initial mutant models (L309P, R339Q, R339W, R377H and G420D) were built using 'Build and Edit Protein' tool in DS by substituting original amino acid residues for the respective mutant. These mutant models were further optimized for their minimum energy confirmation using steepest descent algorithm in DS with a non-bonded lower cut-off distance of 10 Å. Impact of mutations on protein stability was analyzed using 'Calculate Mutation Energy (Stability)' protocol in DS. The protocol calculates the difference in the free energy of folding $\left(\Delta \Delta \mathrm{G}_{\mathrm{mut}}\right)$ between the mutant structure and the wild type protein as follows:

$\Delta \Delta \mathrm{G}_{\mathrm{mut}}=\Delta \Delta \mathrm{Gfold}_{(\text {mutant })}-\Delta \Delta \mathrm{Gfold}_{(\text {wildtype })}$

- where $\Delta \Delta$ Gfold is defined as the free energy difference between the folded and unfolded state of the protein. The unfolded state is modelled as a relaxed protein in extended conformation with the mutated residue in the center.

To further analyse the impact of the mutation on CTCF binding ability to DNA, we first superimposed mutant CTCF models onto the wildtype CTCF model in complex with DNA using Chimera version 1.14. Furthermore, we performed MD simulations of WT and all the mutant models using GROMACS version 4.5.3. The system (CTCF mutant model in complex with DNA) was placed in the center of a cubic box with at least $1 \mathrm{~nm}$ from the box edges. The ions $\left(\mathrm{Na}^{+}\right.$and $\left.\mathrm{Cl}^{-}\right)$were added to the system for neutralizing and preserving at physiological concentration $(0.15 \mathrm{M})$. The protocol consisted of successive rounds of energy minimization, annealing, equilibration, and trajectory 
production in implicit solvent represented by the generalized Born/solvent-accessible surface area model and using a distance cut-off of $10 \AA$ to short-range, non-bonded interactions. Keeping backbone atoms restrained, the protein was relaxed with 50,000 steps of energy minimization, followed by annealing with a $60-300 \mathrm{~K}$ temperature ramp applied over $100 \mathrm{ps}$. In equilibration, the temperature was maintained at $300 \mathrm{~K}$ using Langevin dynamics for 50,000 steps over 100 ps. Production simulations were performed in the isothermal-isobaric ensemble, keeping both the DNA fragment and CTCF unconstrained. Bonds between hydrogen and heavy atoms were constrained at their equilibrium length using LINCS algorithm. Root-mean-square deviation (RMSD), root-meansquare fluctuation (RMSF), secondary structure and interaction energy analyses were carried out using GROMACS for the entire simulation run. All non-bonded interactions for the final poses of CTCF wildtype and mutants in complex with DNA were identified using DS.

\section{List of Abbreviations}

ChIP, chromatin immunoprecipitation; CTCF, CCCTC-binding factor; DMEM, Dulbecco's Modified Eagle Medium; EMSA, electrophoretic mobility shift assay; FACS, fluorescence-activated cell sorting; HA, hemagglutinin; ICR, imprinting control region; MD, molecular dynamics; MTT, 3(4,5-1,2 methylthiazol-2-yl)-2,5-diphenyltetrazolium bromide; PBST, phosphate buffered saline Tween 20; PDB, Protein Data Bank; RMSD, root-mean-square deviation; RMSF, root-mean-square fluctuation; RPMI, Roswell Park Memorial Institute; SNP, single nucleotide polymorphism; TAD, topologically associating domains; TF, transcription factor; WT, wildtype; ZF, zinc finger.

\section{Declarations}

\section{Ethics approval and consent to participate}

Not applicable.

\section{Consent of publication}


Not applicable.

\section{Availability of data and material}

All data generated or analysed during this study are included in this published article (and its supplementary information files). Additionally, PDB files for CTCF ZF WT and mutant conformations have been submitted to Protein Data Bank, accession files: L309P, 1A2B; R339Q, 3C4D; R339W, 5E6F; R377H, 7G8H; G420D, 9I0J.

\section{Competing interests}

The authors declare that they have no competing interests

\section{Funding}

Financial support was provided by Tour de Cure (Scott Canner Research Fellowship) to C.G.B. and for research grants to C.G.B. and J.E.J.R; National Health \& Medical Research Council funding (Investigator Grant \#1177305 and Project Grants \#507776 and \#1128748 to J.E.J.R); Cancer Council NSW project grants (RG11-12, RG14-09, RG20-12) to J.E.J.R. C.G.B. and U.S.; support grants from Cure The Future Foundation and an anonymous foundation. U.S holds a fellowship from the Cancer Institute New South Wales. C.G.M. was supported with grants NCI CA021765 and R35 CA197695. O.W., S.K.G. and K.P.S. acknowledge support from Bundesministerium für Bildung und Forschung (BMBF) grant eBio:MelAutim (01ZX1905B) and European Union's Horizon 2020 research and innovation programme under the Marie Skłodowska-Curie grant agreement No 765274.

\section{Authors' contributions}

C.G.B conceived the study, designed constructs, analysed data and wrote the manuscript. J.E.J.R. reviewed the manuscript, supervised research governance and provided scientific discussion; C.M., P.O'Y., M.A., T.L. and H.F. performed cellular biology experiments; W.K. and T.L. performed ChIP 
and preliminary structural modelling; C.S. performed immunofluorescence; C.G.M provided mutation data; U.S. analysed mutation data and reviewed the manuscript; S.G \& K.P.S \& O.W. performed and reviewed structural modelling, docking and molecular dynamics simulations.

\section{Acknowledgements}

The authors acknowledge The University of Sydney High Performance Computing service at The University of Sydney for providing resources that have contributed to the research data reported within this paper.

\section{Authors' information}

Not applicable 


\section{References}

1. Cerami, E. et al. The cBio cancer genomics portal: an open platform for exploring multidimensional cancer genomics data. Cancer Discov 2, 401-4 (2012).

2. Lawrence, M. S. et al. Mutational heterogeneity in cancer and the search for new cancer-associated genes. Nature 499, 214-8 (2013).

3. Lawrence, M. S. et al. Discovery and saturation analysis of cancer genes across 21 tumour types. Nature 505, 495-501 (2014).

4. Kandoth, C. et al. Mutational landscape and significance across 12 major cancer types. Nature 502, 333-9 (2013).

5. Vogelstein, B. et al. Cancer genome landscapes. Science 339, 1546-58 (2013).

6. Bradner, J. E., Hnisz, D. \& Young, R. A. Transcriptional Addiction in Cancer. Cell 168, 629-643 (2017).

7. Zuber, J. et al. RNAi screen identifies Brd4 as a therapeutic target in acute myeloid leukaemia. Nature 478, 524-8 (2011).

8. Delmore, J. E. et al. BET bromodomain inhibition as a therapeutic strategy to target c-Myc. Cell 146, 904-17 (2011).

9. Dawson, M. A. et al. Inhibition of BET recruitment to chromatin as an effective treatment for MLL-fusion leukaemia. Nature 478, 529-33 (2011).

10. Chapuy, B. et al. Discovery and characterization of super-enhancer-associated dependencies in diffuse large B cell lymphoma. Cancer Cell 24, 777-90 (2013).

11. Vaquerizas, J. M., Kummerfeld, S. K., Teichmann, S. A. \& Luscombe, N. M. A census of human transcription factors: function, expression and evolution. Nat Rev Genet 10, 252-63 (2009).

12. Ravasi, T. et al. An atlas of combinatorial transcriptional regulation in mouse and man. Cell 140, 744-52 (2010).

13. Schmitges, F. W. et al. Multiparameter functional diversity of human $\mathrm{C} 2 \mathrm{H} 2$ zinc finger proteins. Genome Res 26, 1742-1752 (2016). 
14. Najafabadi, H. S. et al. C2H2 zinc finger proteins greatly expand the human regulatory lexicon. Nat Biotechnol 33, 555-62 (2015).

15. Nakahashi, H. et al. A Genome-wide Map of CTCF Multivalency Redefines the CTCF Code. Cell Rep 3, 1678-89 (2013).

16. Phillips, J. E. \& Corces, V. G. CTCF: master weaver of the genome. Cell 137, 1194-211 (2009).

17. Merkenschlager, M. \& Odom, D. T. CTCF and Cohesin: Linking Gene Regulatory Elements with Their Targets. Cell 152, 1285-97 (2013).

18. Sofueva, S. et al. Cohesin-mediated interactions organize chromosomal domain architecture. EMBO J 32, 3119-29 (2013).

19. Zuin, J. et al. Cohesin and CTCF differentially affect chromatin architecture and gene expression in human cells. Proc Natl Acad Sci U A 111, 996-1001 (2014).

20. Dixon, J. R. et al. Topological domains in mammalian genomes identified by analysis of chromatin interactions. Nature 485, 376-80 (2012).

21. Nora, E. P. et al. Spatial partitioning of the regulatory landscape of the X-inactivation centre. Nature 485, 381-5 (2012).

22. Seitan, V. C. et al. Cohesin-based chromatin interactions enable regulated gene expression within preexisting architectural compartments. Genome Res 23, 2066-77 (2013).

23. Vietri Rudan, M. et al. Comparative Hi-C Reveals that CTCF Underlies Evolution of Chromosomal Domain Architecture. Cell Rep 10, 1297-309 (2015).

24. Nora, E. P. et al. Targeted Degradation of CTCF Decouples Local Insulation of Chromosome Domains from Genomic Compartmentalization. Cell 169, 930-944 (2017).

25. Tang, Z. et al. CTCF-Mediated Human 3D Genome Architecture Reveals Chromatin Topology for Transcription. Cell 163, 1611-27 (2015).

26. Lupiáñez, D. G. et al. Disruptions of Topological Chromatin Domains Cause Pathogenic Rewiring of Gene-Enhancer Interactions. Cell 161, 1012-25 (2015). 
27. Guo, Y. et al. CRISPR Inversion of CTCF Sites Alters Genome Topology and Enhancer/Promoter Function. Cell 162, 900-10 (2015).

28. Narendra, V. et al. Transcription. CTCF establishes discrete functional chromatin domains at the Hox clusters during differentiation. Science 347, 1017-21 (2015).

29. Arzate-Mejía, R. G., Recillas-Targa, F. \& Corces, V. G. Developing in 3D: the role of CTCF in cell differentiation. Development 145, 1-13 (2018).

30. Moore, J. M. et al. Loss of maternal CTCF is associated with peri-implantation lethality of Ctcf null embryos. PLoS One 7, e34915 (2012).

31. Bailey, C. G. et al. CTCF Expression is Essential for Somatic Cell Viability and Protection Against Cancer. Int J Mol Sci 19, 12-31 (2018).

32. Rasko, J. E. et al. Cell growth inhibition by the multifunctional multivalent zinc-finger factor CTCF. Cancer Res 61, 6002-7 (2001).

33. Tiffen, J. C. et al. The cancer-testis antigen BORIS phenocopies the tumor suppressor CTCF in normal and neoplastic cells. Int J Cancer 133, 1603-13 (2013).

34. Filippova, G. N. et al. Tumor-associated zinc finger mutations in the CTCF transcription factor selectively alter its DNA-binding specificity. Cancer Res 62, 48-52 (2002).

35. Kandoth, C. et al. Integrated genomic characterization of endometrial carcinoma. Nature 497, $67-73$ (2013).

36. Zighelboim, I. et al. High Frequency Strand Slippage Mutations in CTCF in MSI-Positive Endometrial Cancers. Hum Mutat 1, 63-5 (2014).

37. Walker, C. J. et al. Patterns of CTCF and ZFHX3 Mutation and Associated Outcomes in Endometrial Cancer. J Natl Cancer Inst 107, 11-20 (2015).

38. Marshall, A. D. et al. CTCF genetic alterations in endometrial carcinoma are pro-tumorigenic. Oncogene 36, 4100-4110 (2017).

39. Mullighan, C. G. et al. CREBBP mutations in relapsed acute lymphoblastic leukaemia. Nature 471, 235-9 (2011). 
40. Liu, Y. et al. The genomic landscape of pediatric and young adult T-lineage acute lymphoblastic leukemia. Nat Genet 49, 1211-1218 (2017).

41. Ding, L.-W. W. et al. Mutational Landscape of Pediatric Acute Lymphoblastic Leukemia. Cancer Res 77, 390-400 (2017).

42. Yoshida, K. et al. The landscape of somatic mutations in Down syndrome-related myeloid disorders. Nat Genet 45, 1293-9 (2013).

43. Hashimoto, H. et al. Structural Basis for the Versatile and Methylation-Dependent Binding of CTCF to DNA. Mol Cell 66, 711-720 (2017).

44. Zhang, J. et al. Key pathways are frequently mutated in high risk childhood acute lymphoblastic leukemia: a report from the Children's Oncology Group. Blood 118, 3080-7 (2011).

45. Li, Y. et al. The structural basis for cohesin-CTCF-anchored loops. Nature 578, 472-476 (2020).

46. Filippova, G. N. et al. An exceptionally conserved transcriptional repressor, CTCF, employs different combinations of zinc fingers to bind diverged promoter sequences of avian and mammalian c-myc oncogenes. Mol Cell Biol 16, 2802-13 (1996).

47. Quitschke, W. W., Taheny, M. J., Fochtmann, L. J. \& Vostrov, A. A. Differential effect of zinc finger deletions on the binding of CTCF to the promoter of the amyloid precursor protein gene. Nucleic Acids Res 28, 3370-8 (2000).

48. Renda, M. et al. Critical DNA binding interactions of the insulator protein CTCF: a small number of zinc fingers mediate strong binding, and a single finger-DNA interaction controls binding at imprinted loci. J Biol Chem 282, 33336-45 (2007).

49. Yin, M. et al. Molecular mechanism of directional CTCF recognition of a diverse range of genomic sites. Cell Res 27, 1365-1377 (2017).

50. Miller, J., McLachlan, A. D. \& Klug, A. Repetitive zinc-binding domains in the protein transcription factor IIIA from Xenopus oocytes. EMBO J 4, 1609-14 (1985).

51. Siggers, T., Reddy, J., Barron, B. \& Bulyk, M. L. Diversification of transcription factor paralogs via noncanonical modularity in C2H2 zinc finger DNA binding. Mol Cell 55, 640-8 (2014). 
52. Garton, M. et al. A structural approach reveals how neighbouring $\mathrm{C} 2 \mathrm{H} 2$ zinc fingers influence DNA binding specificity. Nucleic Acids Res 43, 9147-57 (2015).

53. Kung, J. T. et al. Locus-Specific Targeting to the X Chromosome Revealed by the RNA Interactome of CTCF. Mol Cell 57, 361-75 (2015).

54. Sun, S. et al. Jpx RNA Activates Xist by Evicting CTCF. Cell 153, 1537-1551 (2013).

55. Saldaña-Meyer, R. et al. RNA Interactions Are Essential for CTCF-Mediated Genome Organization. Mol Cell 76, 412-422 (2019).

56. Pant, V. et al. Mutation of a single CTCF target site within the H19 imprinting control region leads to loss of Igf2 imprinting and complex patterns of de novo methylation upon maternal inheritance. Mol Cell Biol 24, 3497-504 (2004).

57. Ohlsson, R., Lobanenkov, V. \& Klenova, E. Does CTCF mediate between nuclear organization and gene expression? Bioessays 32, 37-50 (2010).

58. Rao, S. S. P. et al. A 3D map of the human genome at kilobase resolution reveals principles of chromatin looping. Cell 159, 1665-80 (2014).

59. Gómez-Marín, C. et al. Evolutionary comparison reveals that diverging CTCF sites are signatures of ancestral topological associating domains borders. Proc Natl Acad Sci U A 112, 7542-7 (2015).

60. Ong, C.-T. T. \& Corces, V. G. CTCF: an architectural protein bridging genome topology and function. Nat Rev Genet 15, 234-46 (2014).

61. Rao, S. S. P. et al. Cohesin Loss Eliminates All Loop Domains. Cell 171, 305-320 (2017).

62. Hanssen, L. L. P. et al. Tissue-specific CTCF-cohesin-mediated chromatin architecture delimits enhancer interactions and function in vivo. Nat Cell Biol 19, 952-961 (2017).

63. Hu, J. et al. Chromosomal Loop Domains Direct the Recombination of Antigen Receptor Genes. Cell 163, 947-59(2015).

64. Flavahan, W. A. et al. Insulator dysfunction and oncogene activation in IDH mutant gliomas. Nature 529, 110-4 (2016). 
bioRxiv preprint doi: https://doi.org/10.1101/2021.01.08.425848; this version posted January 8,2021 . The copyright holder for this preprint

(which was not certified by peer review) is the author/funder, who has granted bioRxiv a license to display the preprint in perpetuity. It is made available under aCC-BY-NC-ND 4.0 International license.

65. Weischenfeldt, J. et al. Pan-cancer analysis of somatic copy-number alterations implicates IRS4 and IGF2 in enhancer hijacking. Nat Genet 49, 65-74 (2017).

66. Hnisz, D. et al. Activation of proto-oncogenes by disruption of chromosome neighborhoods.

Science 351, 1454-1458 (2016). 


\section{Figure Legends}

Figure 1 Distribution and impact of CTCF somatic mutations in cancer. (A) The landscape of somatic mutations (above) and SNPs (below) occurring in CTCF: the distribution and frequency within the coding region is shown, recurrent somatic mutations (occurring $\geq 10$ times) are labelled. For a curated list of non-redundant CTCF mutations from cancer genome sequencing studies (TCGA, COSMIC) and published studies see Supplementary Table 1. CTCF mutation type (B); and tissue distribution (C) are shown; $n=$ total number of mutations. (D) Analysis of cancer-related somatic missense variants and missense SNPs occurring in each domain of CTCF. The expected occurrence was calculated from the total number with the proportion of missense variants expected in each domain if they were evenly distributed. The observed/expected ratio confirms if there is a deenrichment $(<1.0)$ or an enrichment $(>1.0)$ of non-synonymous changes. (E) Frequency of somatic missense mutations occurring in specific ZFs of CTCF, the mean for all ZFs is shown (dotted line). (F) Sequence logo of all 11 aligned CTCF ZFs; numbers $(-6$ to +6$)$ indicate co-ordinates within the DNA-binding portion of the ZF. Similar amino acids are coloured: black - hydrophobic (G, A, V, I, L, P, W, F, M); green - polar $(\mathrm{S}, \mathrm{T}, \mathrm{Y}, \mathrm{C})$; purple - polar amide $(\mathrm{Q}, \mathrm{N})$; blue - basic $(\mathrm{K}, \mathrm{R}, \mathrm{H})$; and red - acidic $(\mathrm{D}, \mathrm{E})$. The height of each amino acid residue is proportional to its observed frequency. The overall height of each letter 'stack' is proportional to the sequence conservation, shown in bits (G) Frequency of missense somatic mutations at each ZF position; the mean for all ZFs is shown (dotted line). Data represent the mean $\pm \mathrm{SD}$ with statistical analysis performed using the Chi-square test $(*, \mathrm{p}<0.05 ; * *, \mathrm{p}<0.01 ; * * * *, \mathrm{p}<0.0001)$.

Figure 2 Functional impacts of CTCF ZF missense mutations. (A) Published and unpublished missense CTCF mutations (red circles) occurring in acute lymphoblastic leukaemia (L309P, R339Q, R337H, G420D - highlighted in black) superimposed on a C2H2 ZF structure: $\mathrm{C}=$ cysteine, $\mathrm{H}=$ histidine, $\mathrm{Zn}=\mathrm{Zn}^{2+}$ ion; $\mathrm{R} 339 \mathrm{~W}$ (underlined) is a previously characterized change-of-function mutation used as a control. The Polyphen score for each mutation is indicated. Numbers $(-6-+6)$ 
indicate co-ordinates within the DNA-binding portion of the ZF; residues directly contacting DNA at positions $-1,+2,+3$ and +6 are indicated (white ring). (B) Western blot of WT and mutant CTCF expression in transduced K562 cells; anti-HA antibody detects ectopic CTCF, CTCF antibody detects total CTCF; GAPDH is a loading control; size markers (not shown) indicate MW in kDa; vertical dashed line indicates WT sample run on same blot but relocated for clarity. (C) Immunofluorescence of HA-tagged WT and mutant CTCF in K562 cells using anti-HA antibody, scale bar=5 $\mu$ m. (D-E) Functional assays of CTCF mutants in K562 cells including: (D) MTT proliferation; and (E) colony forming assay in Methocult. Data represent the mean \pm s.e.m for 3 experiments each performed in triplicate. Statistical analysis was performed using Mann-Whitney U-test (ns = not significant; *, $p<0.05 ; * *, p<0.01 ; * * *, p<0.001 ; * * * *, p<0.0001)$.

Figure 3 Differential gene regulatory activities exhibited by ZF-mutant CTCF. (A) Schematic of the lentivector plasmid used to measure WT and mutant CTCF gene regulatory activity. Predicted CTCF binding sites on both sense and antisense strands are represented by horizontal lines respectively. (B-D) Control (eGFP-alone), CTCF WT or mutant-containing lentivector plasmids were transfected into HEK293T cells for 48 h. (B) Representative Western blots (of 3 replicates) indicating ectopic (HA-tagged) CTCF, total CTCF and GAPDH loading control after transfection of HEK293T cells. (C) Densitometry of ectopic and endogenous CTCF expression normalised to GAPDH, shown relative to WT CTCF set as 1.0. (D) GFP mean fluorescence intensity (MFI) detected after $48 \mathrm{~h}$ and normalised to eGFP empty vector control set as 1.0. Data represent the mean \pm s.e.m for 3 experiments each performed in triplicate except for the Western blots which are only single replicates. Statistical analysis was performed using Mann-Whitney U-test (ns = not significant; ${ }^{*}, p<0.05$; ${ }^{*}, p<0.01 ; * * *$, $p<0.001 ; * * * *, p<0.0001)$.

Figure 4 Differential DNA binding exhibited by ZF-mutant CTCF. ChIP-PCR of HA-tagged WT and mutant CTCF expressed in K562 cells; L = 100 bp ladder, input is total genomic DNA before 
ChIP, Control = eGFP empty vector. Diverse CTCF sites were examined: including (A) archetypal CTCF sites; (B) the $C$-MYC locus; (C) enhancers; (D) insulators; and (E) TAD boundaries. Where relevant, the genomic distance from the TSS is indicated in brackets. The $S L C 7 A 1$ androgen response element (ARE) was used a negative control for CTCF binding. See Supplementary Table 4 listing references for known CTCF sites.

Figure 5 Homology modelling of CTCF ZF mutations. (A) CTCF ZF residues impacted by somatic mutation are depicted on the crystal structure model of ZFs 2-7 in association with DNA. Zinc molecules are shown as grey spheres. (B) The effect of mutation on the binding energy: the change in minimum free energy $(\Delta \Delta \mathrm{G})$ for WT and mutant CTCF ZF structure in the DNA-bound state. (C - G) Overlay images of the normal (WT, grey) and mutant (blue) residues superimposed on the CTCF crystal structure: (C) L309P (L grey, P dark blue); (D) R339Q (R grey, Q cyan); (E) R339W (R grey, W green); (F) R377H (R grey, H magenta); (G) G420D (G grey, D red). Dashed lines indicate hydrogen bond pairing: old (grey) \& new (green). DNA bases and their position relative to the 5' end of the CTCF consensus are shown.

Figure 6 Impact of mutation on CTCF ZF domain conformational stability revealed by MD simulations. (A-B) Root-mean-square deviation (RMSD) measurements were calculated from the position differences of backbone atoms in the native (WT) and various mutant conformations. (A) Trajectories of all 5 mutants and WT CTCF are displayed over a 10 ns time span, measured at 2 ps intervals. (B) Violin plots of all RMSD measurements (0.000 -10.000 ns, 5001 in total). In each plot, the solid black line indicates median and dashed coloured lines indicate quartiles. (C) Root-meansquare fluctuation (RMSF) measurements were obtained for all residues $(n=173)$ at each time point for the WT and mutant structures. In each plot, the mean \pm SD is shown. In (B) \& (C) the Wilcoxon matched-pairs signed rank test was applied to all paired measurements (ns = not significant; *, 
$p<0.05 ; * *, p<0.01 ; * * * *, p<0.0001$ ). (D) The net change in distance (in $\AA$ ) of the centre of mass (centroid) of the associated ZF domain from DNA compared to WT CTCF.

\section{Supplementary Figure Legends}

\section{Supplementary Figure 1 Predicted phenotypes of missense CTCF SNPs and somatic mutations.}

(A) The mean Polyphen score of missense SNPs and somatic mutations in CTCF is shown. (B) Proportion of the transition and transversion mutations for SNPs and somatic missense mutations in CTCF; ratio in brackets. Data represent the mean \pm SD with statistical analysis performed using Mann-Whitney U-test (A) or Chi-square test (B) $(* * * *, p<0.0001)$.

Supplementary Figure 2 WT and mutant CTCF expression in K562 cells. Flow cytometry of eGFP expression achieved from transduction of HA-tagged WT and mutant CTCF lentiviral vectors in K562 cells. Cells were lysed for immunoblot (Figure 2B), prepared for immunofluorescence (Figure 2C) and subjected to formaldehyde cross-linking for ChIP (Figure 4).

Supplementary Figure 3 Time evolution of secondary structure in WT and mutant CTCF ZF structures. The Dictionary of Secondary Structure of Proteins (DSSP) classification of secondary structure was calculated for all amino acid residues over the 10 ns time course: (A) WT; (B) L309P; (C) R339Q; (D) R339W; (E) R377H; and (F) G420D. Arrows indicate the position of each mutation.

Supplementary Figure 4 Flexibility of CTCF ZFs before and after mutation measured by MD simulations. (A-D) Backbone RMSF values during the MD simulations for mutant CTCF ZFs 
bioRxiv preprint doi: https://doi.org/10.1101/2021.01.08.425848; this version posted January 8,2021 . The copyright holder for this preprint (which was not certified by peer review) is the author/funder, who has granted bioRxiv a license to display the preprint in perpetuity. It is made available under aCC-BY-NC-ND 4.0 International license.

compared to WT, spanning their associated ZF domain; dotted vertical line indicates position of mutation. (A) L309P; ZF2 (B) R339Q \& R339W; ZF3 (C) R377H; ZF4 \& 5, (D) G420D; ZF6. 
Bailey et al Figure 1

A

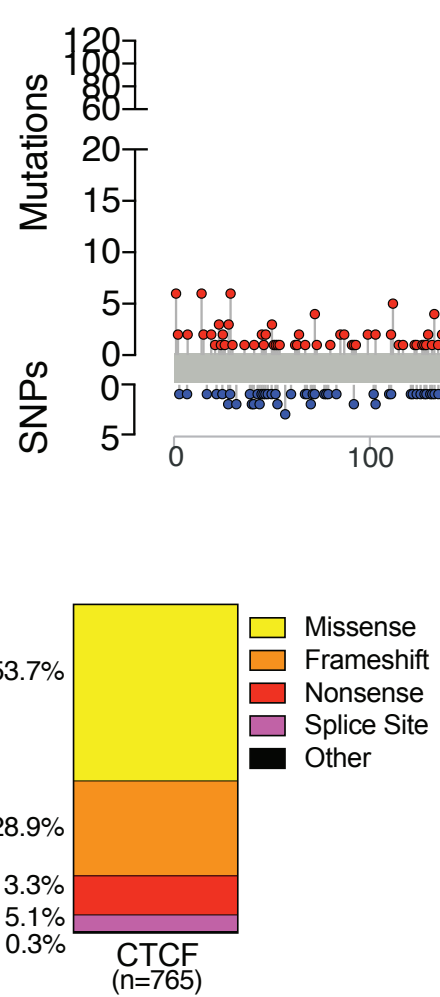

E

B

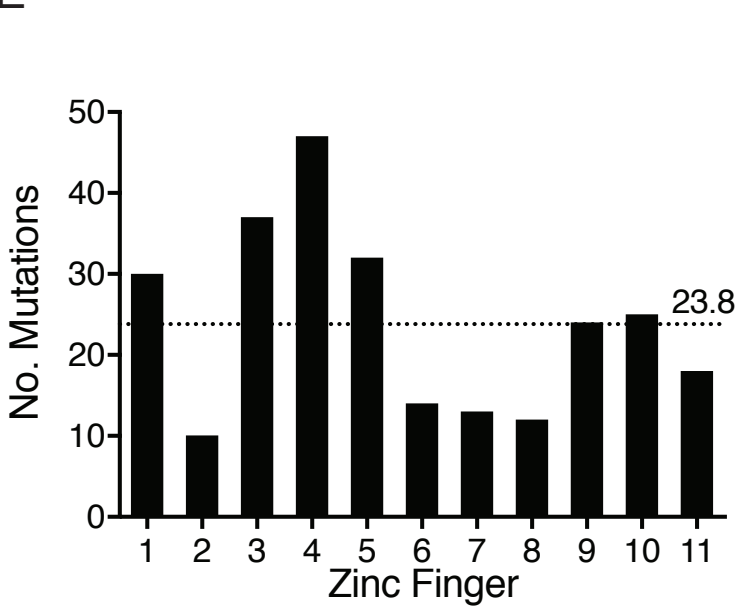

T204f*26 -T204fs*18

CTCF

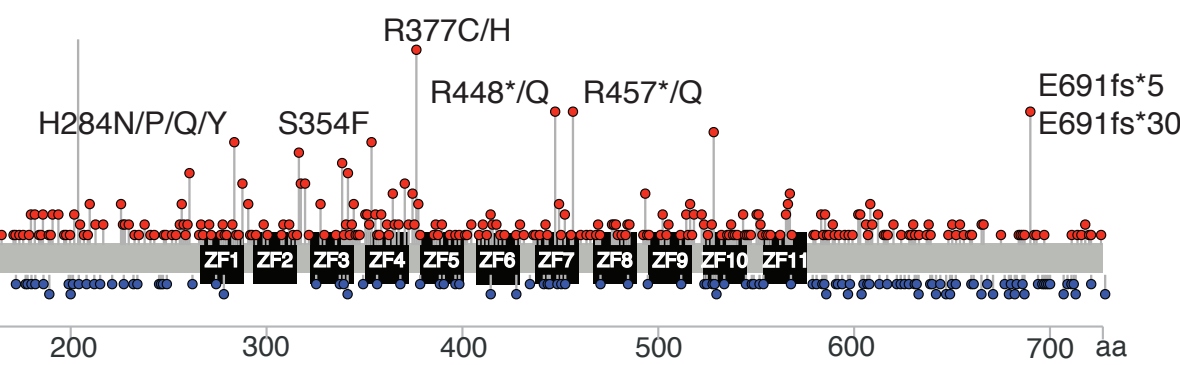

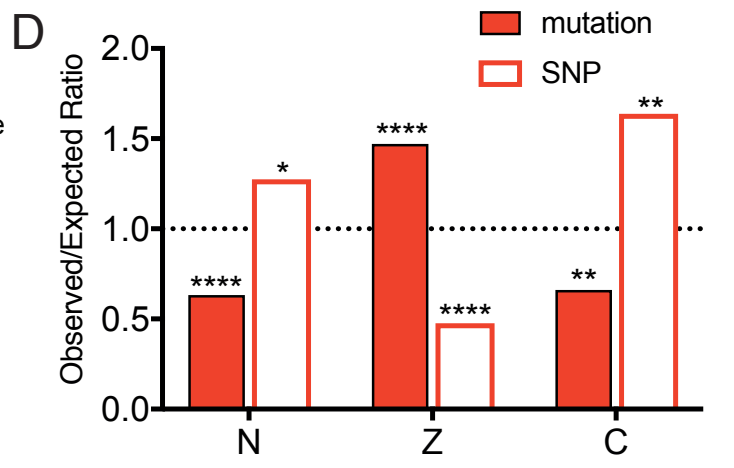

$\mathrm{F}$

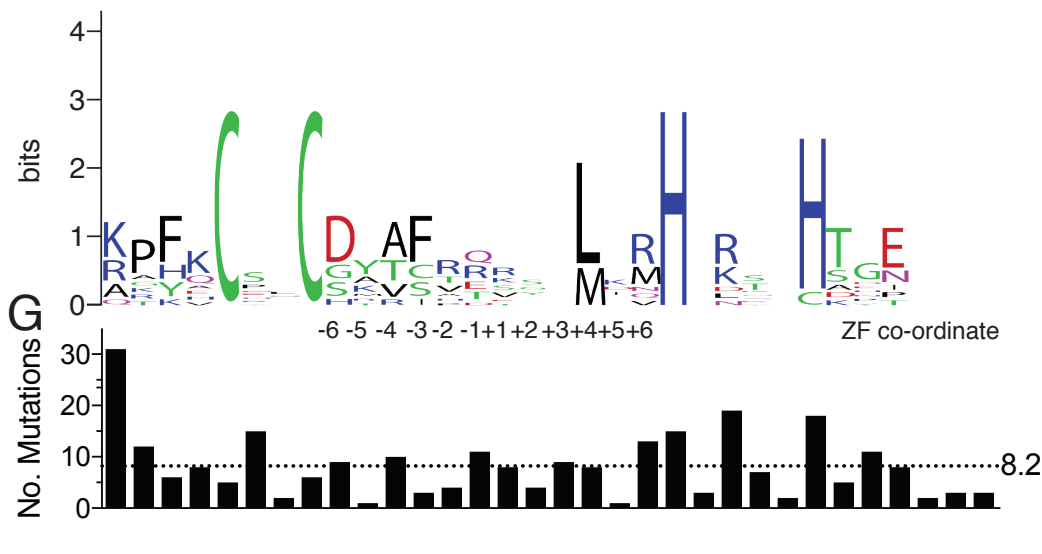

Zinc Finger Position 
A

C
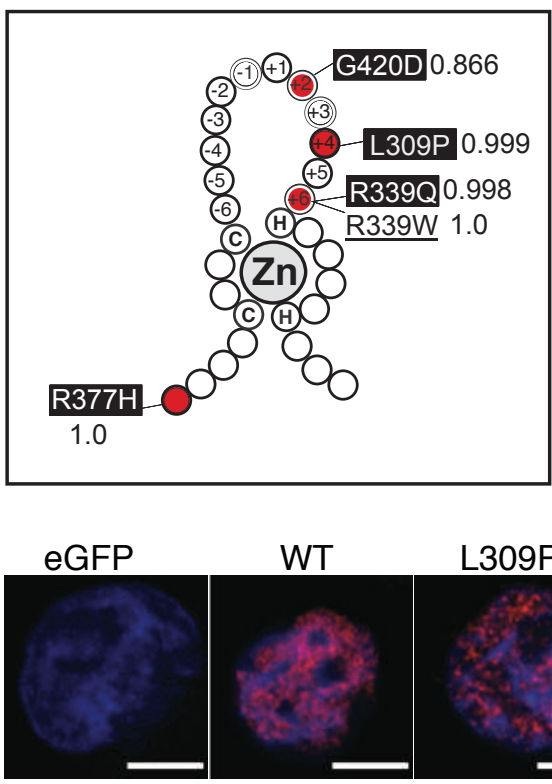

WT

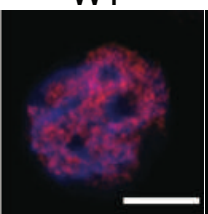

L309P

$\mathrm{D}$

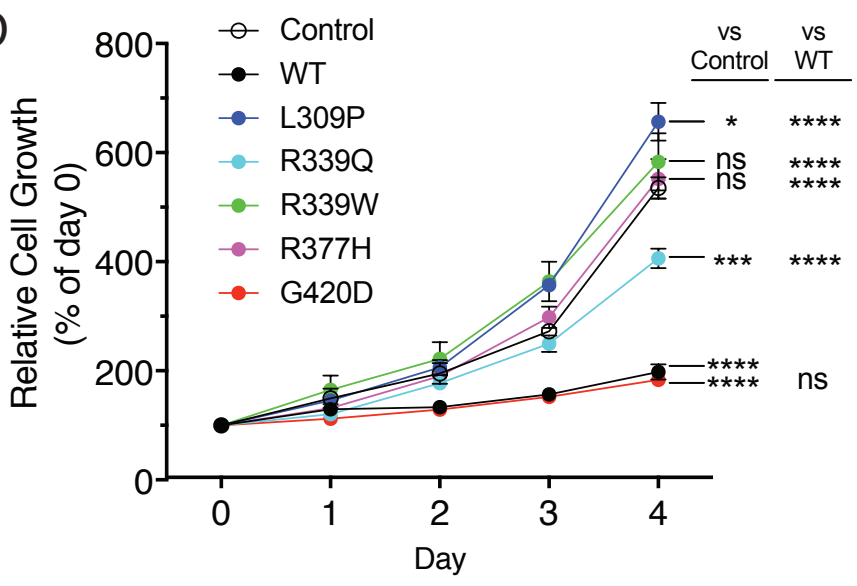

B

CTCF

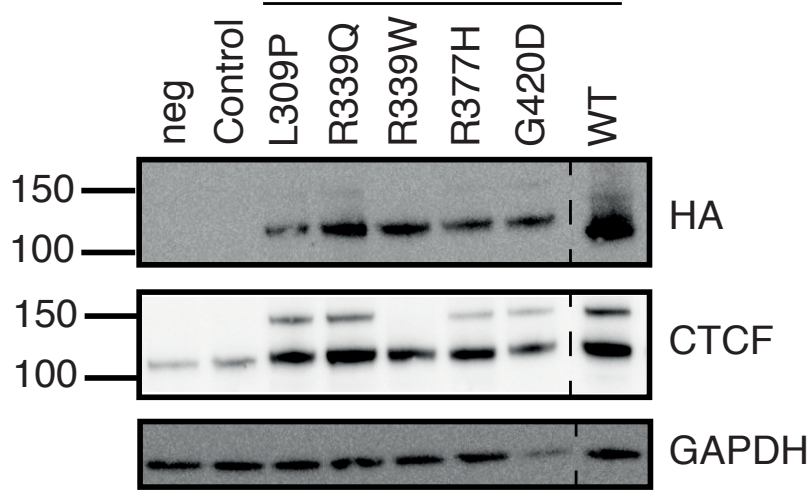

R339Q

R339W

$\mathrm{R} 377 \mathrm{H}$

G420D
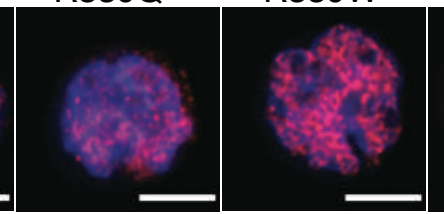

E

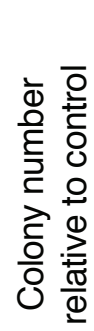

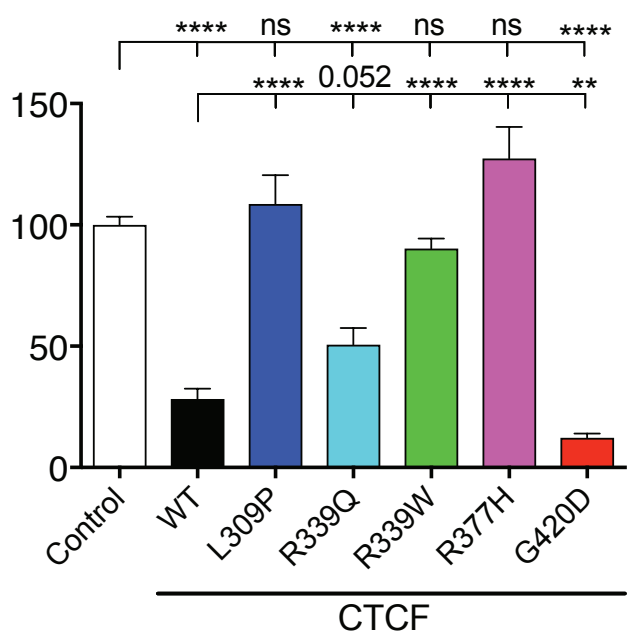


A

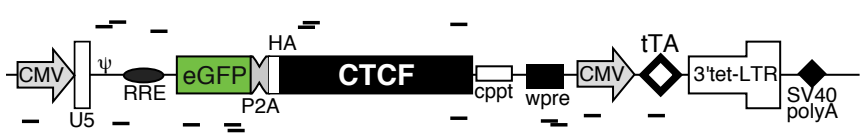

C

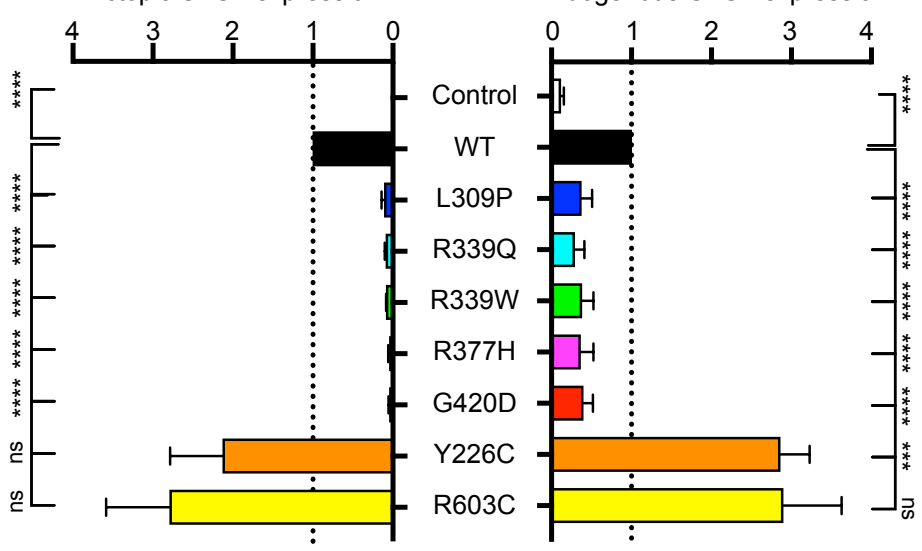

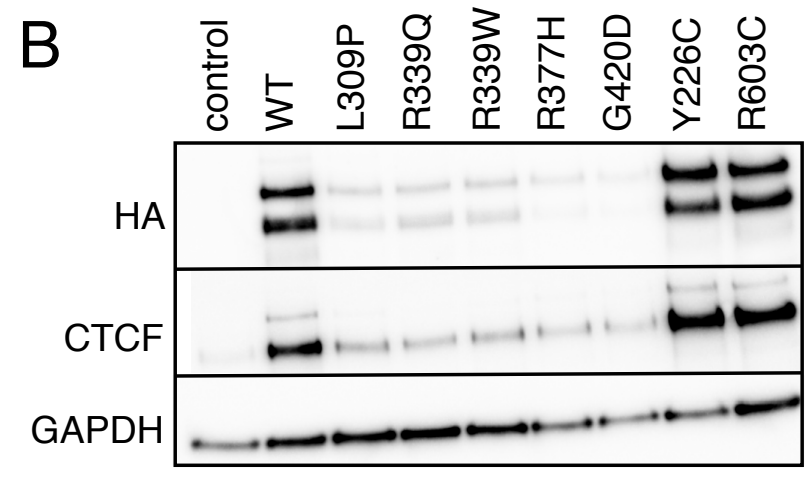

$\mathrm{D}$

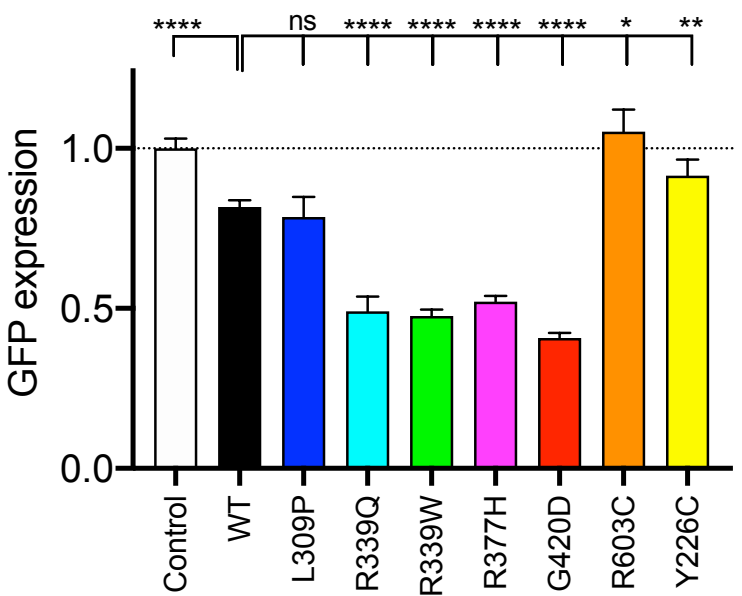


Bailey et al Figure 4

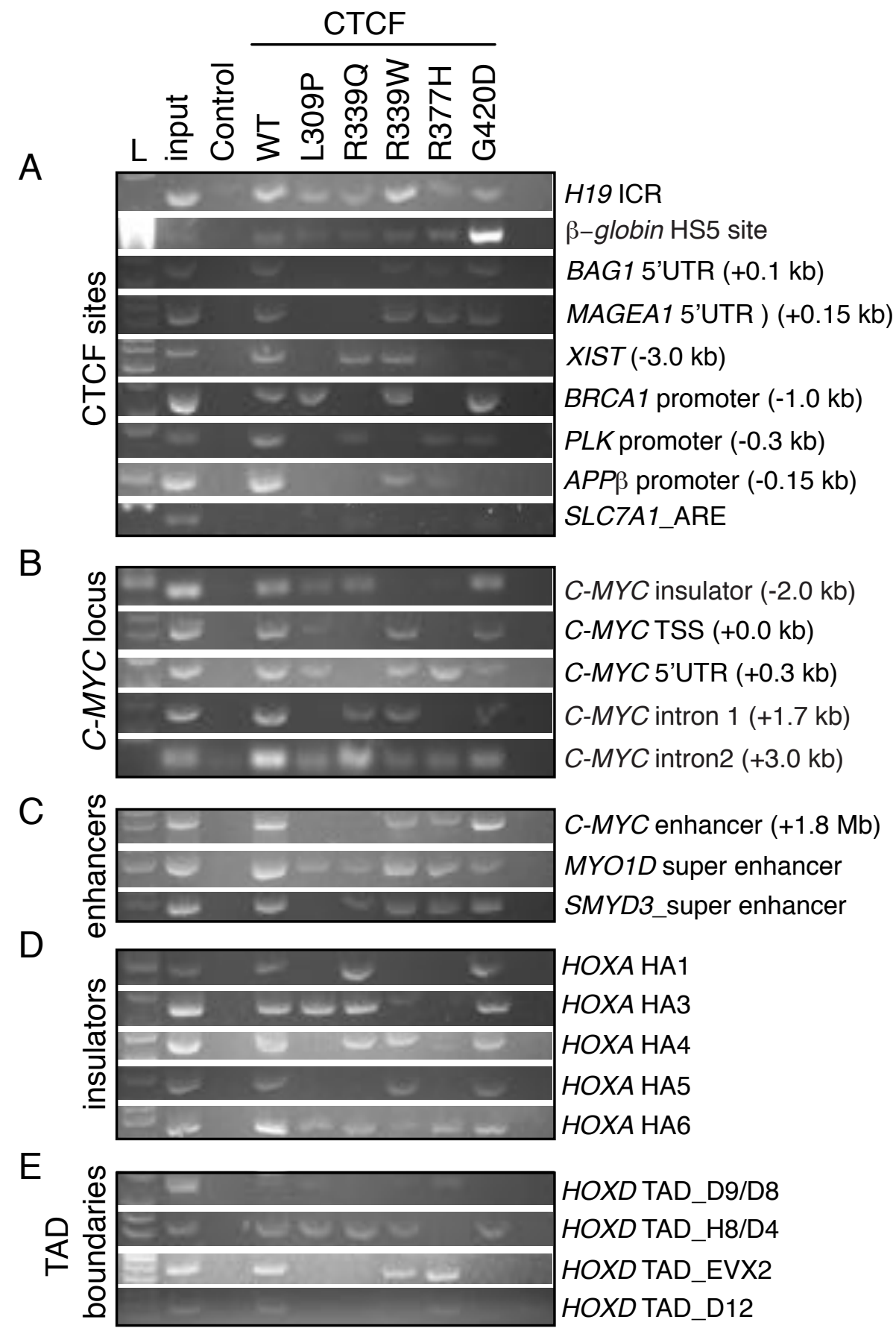


bioRxiv preprint doi: https://doi.org/10.1101/2021.01.08.425848; this version posted January 8, 2021. The copyright holder for this preprint

(which was not certified by peer review) is the author/funder, who has granted bioRxiv a license to display the preprint in perpetuity. It is made available under aCC-BY-NC-ND 4.0 International license.

Bailey et al Figure 5

A

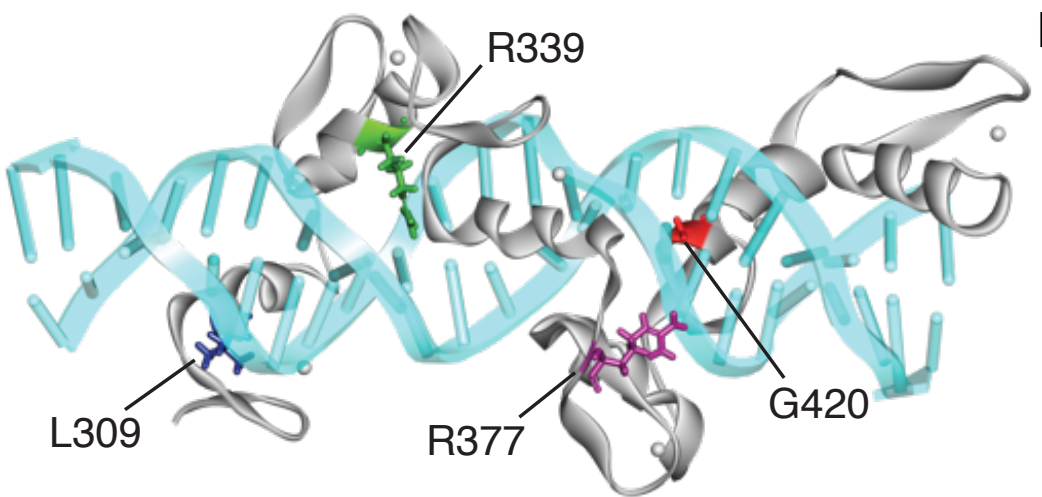

$B$

C L309P

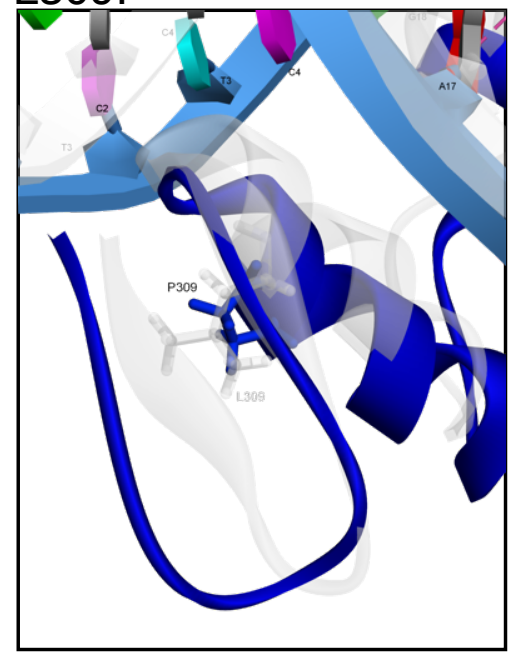

$\mathrm{F} \mathrm{R} 377 \mathrm{H}$

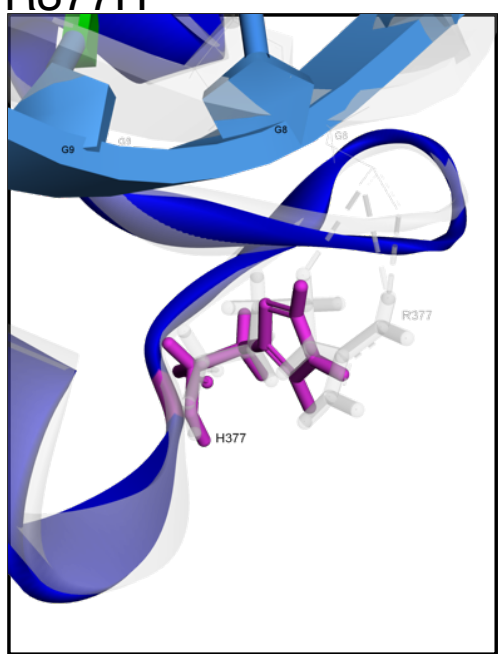

D R339Q

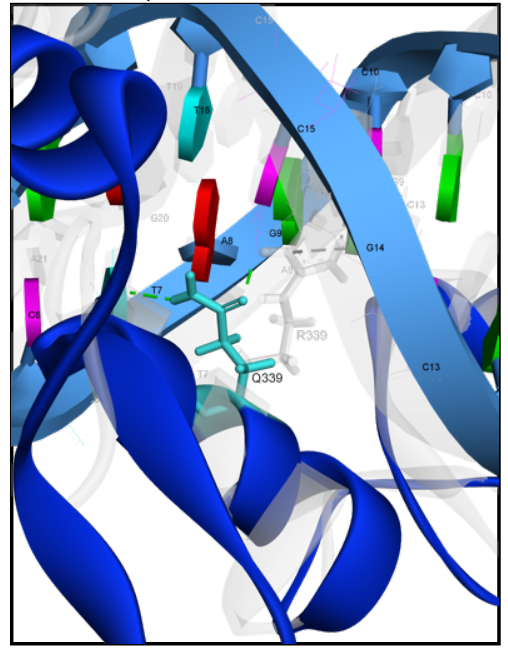

\begin{tabular}{|l|c|c|}
\hline Mutation Site & $\begin{array}{c}\text { Mutation Energy } \\
(\Delta \Delta \mathrm{G}, \mathrm{Kcal} / \mathrm{mol})\end{array}$ & Effect * $^{*}$ \\
\hline L309P & 12.05 & Destabilizing \\
R339Q & 6.87 & Destabilizing \\
R339W & 5.00 & Destabilizing \\
R377H & 5.64 & Destabilizing \\
G420D & 1.91 & Destabilizing \\
\hline
\end{tabular}

E R339W

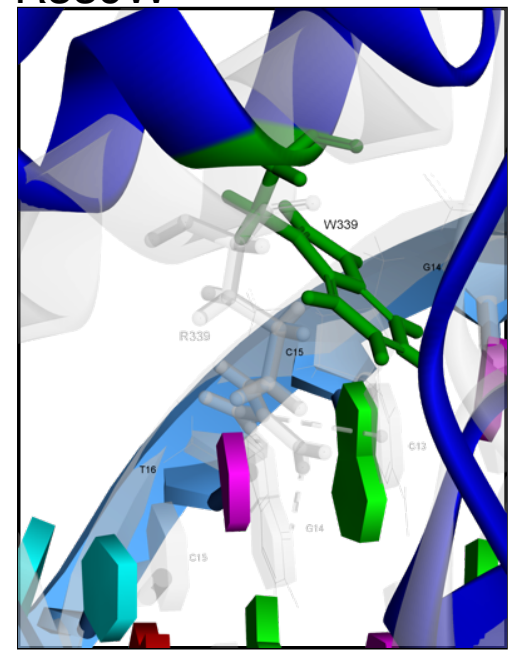

G G420D

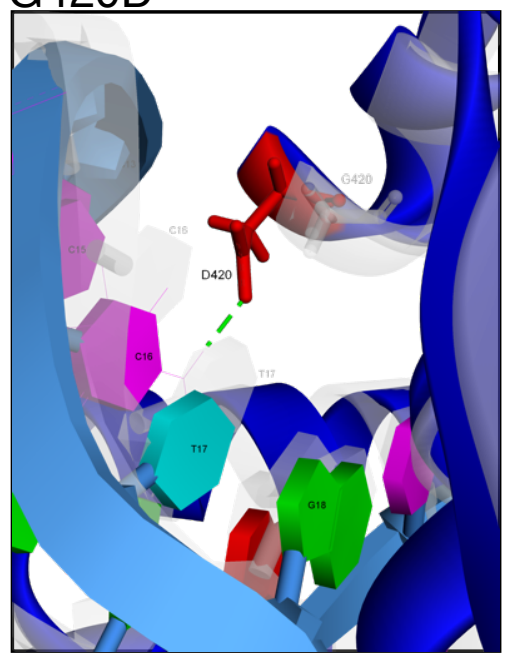


A

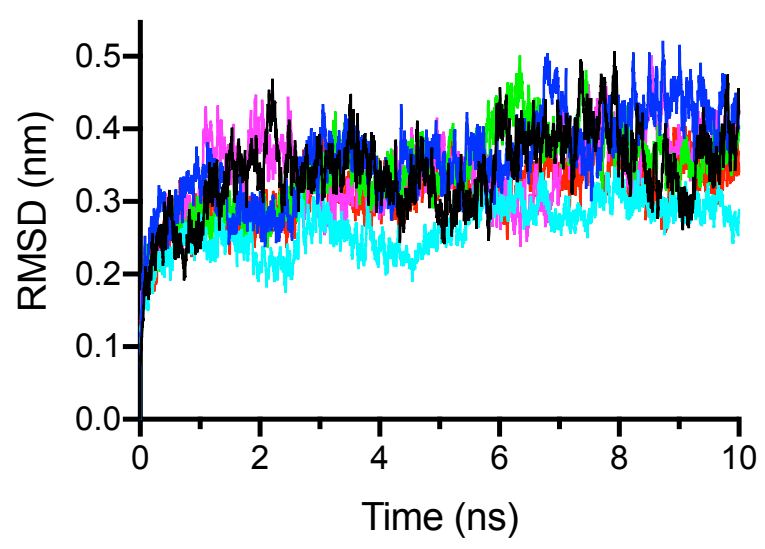

C

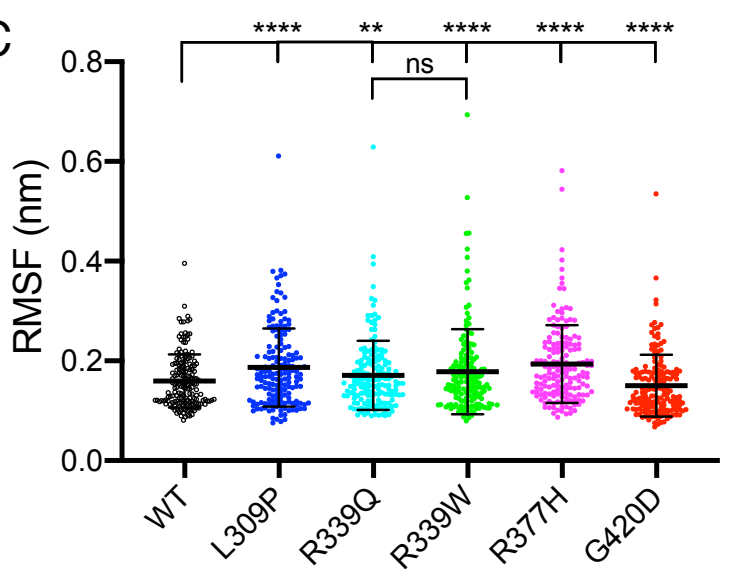

B
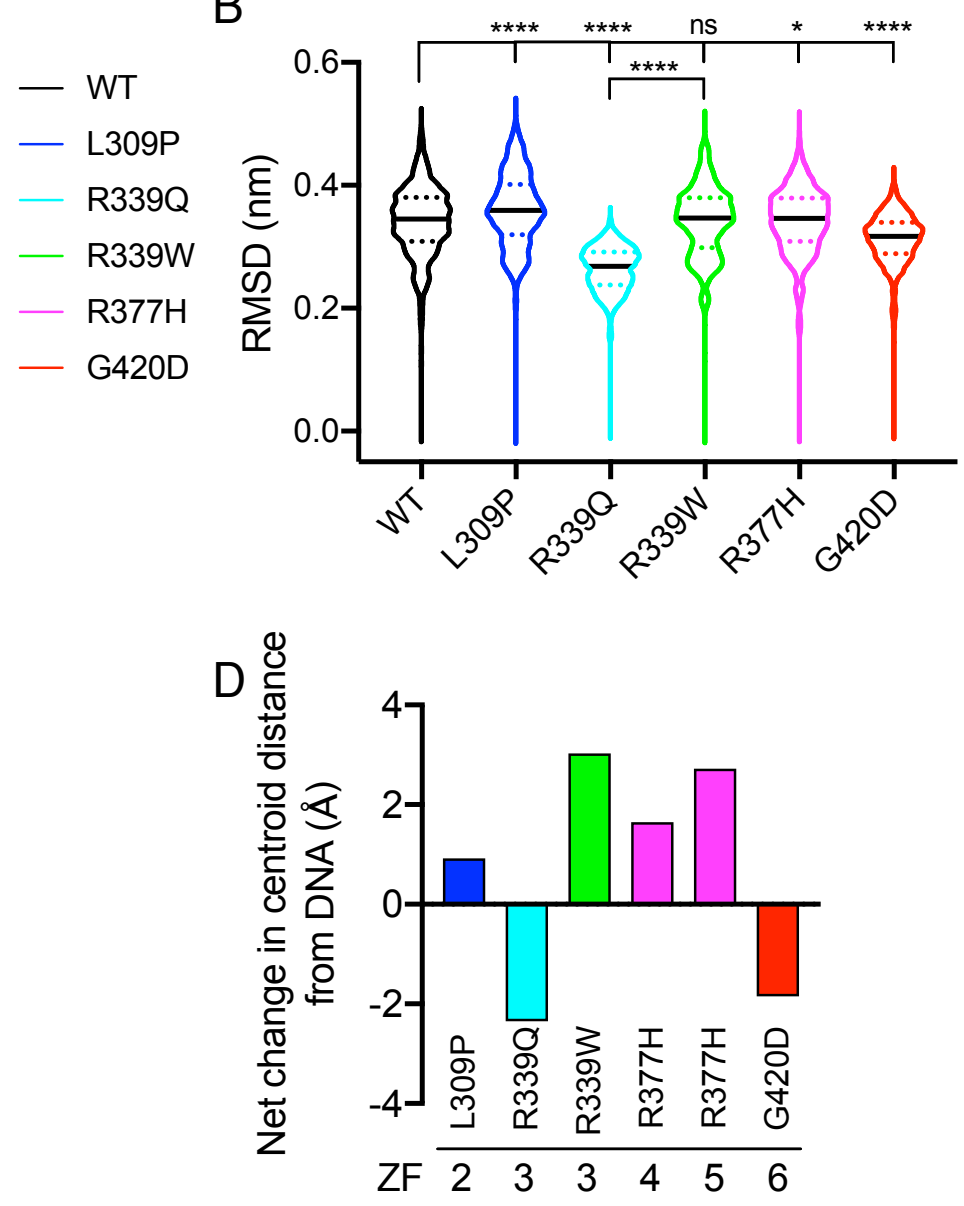


\section{Bailey et al Supplementary Figure_1}

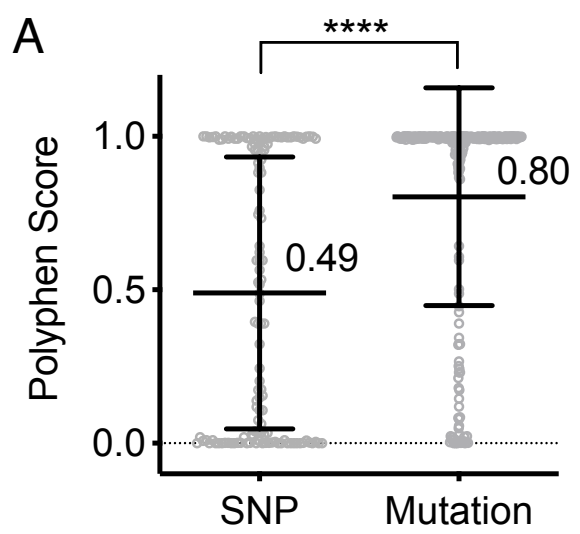

B

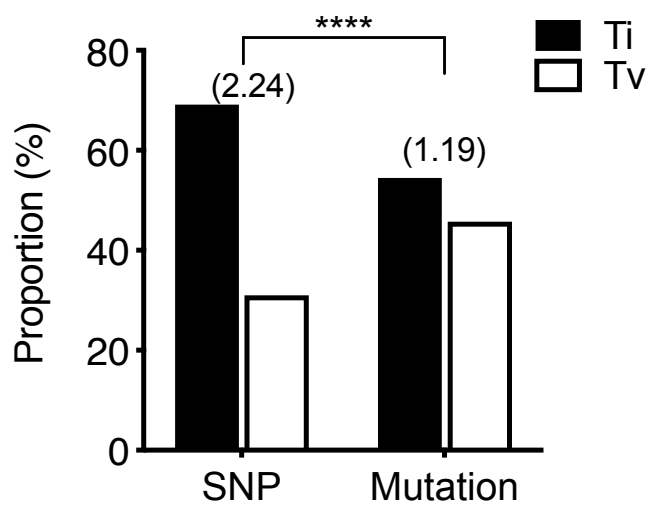


Bailey et al Supplementary Figure 2

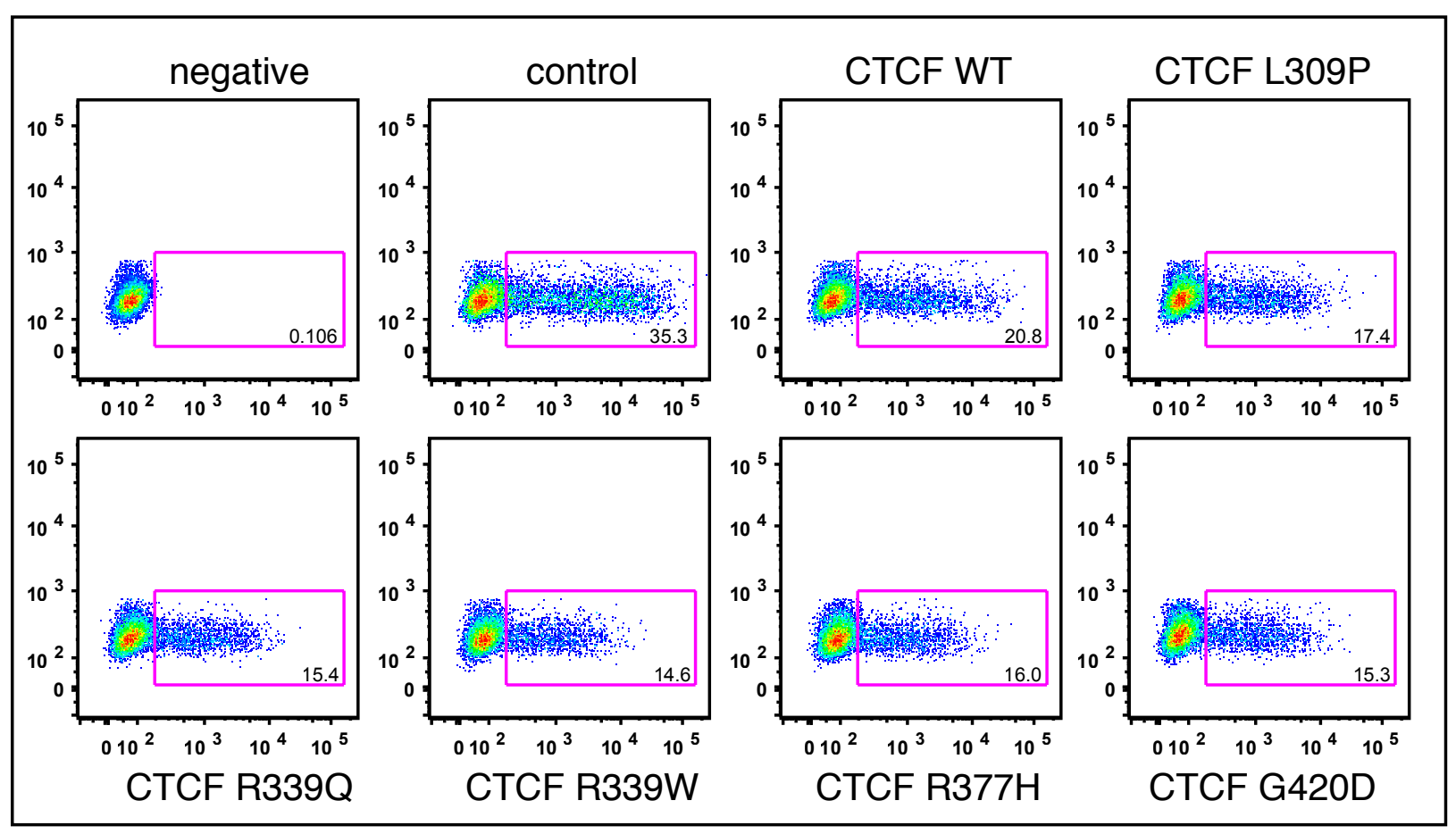


bioRxiv preprint doi: https://doi.org/10.1101/2021.01.08.425848; this version posted January 8, 2021. The copyright holder for this preprint

(which was not certified by peer review) is the author/funder, who has granted bioRxiv a license to display the preprint in perpetuity. It is made available under aCC-BY-NC-ND 4.0 International license.

Bailey et al Supplementary Figure 3

A

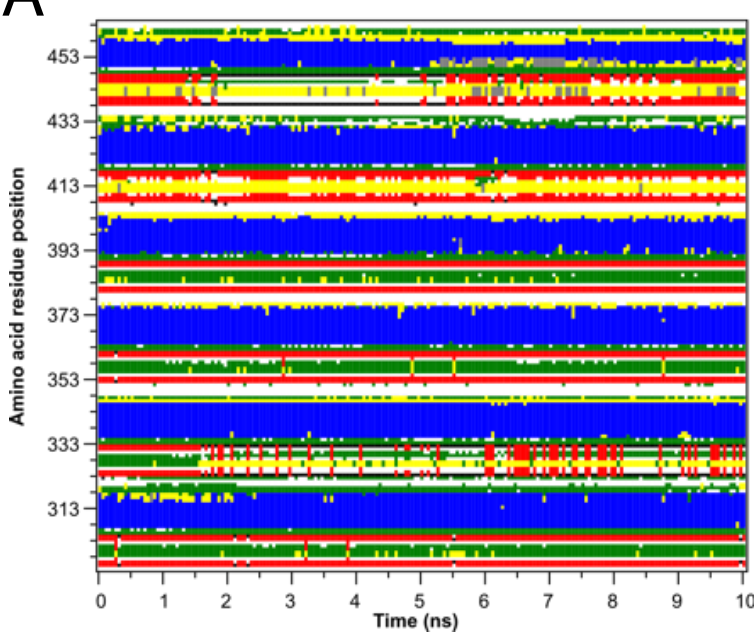

\section{C}

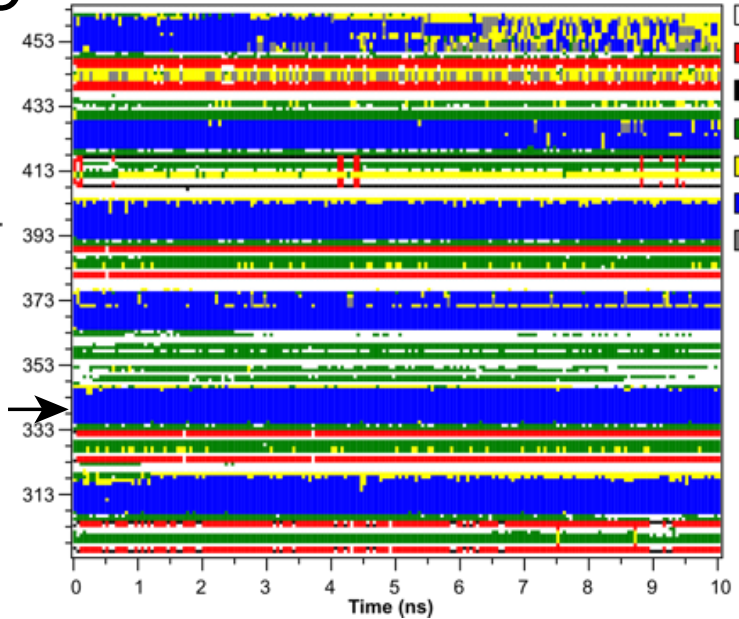

E

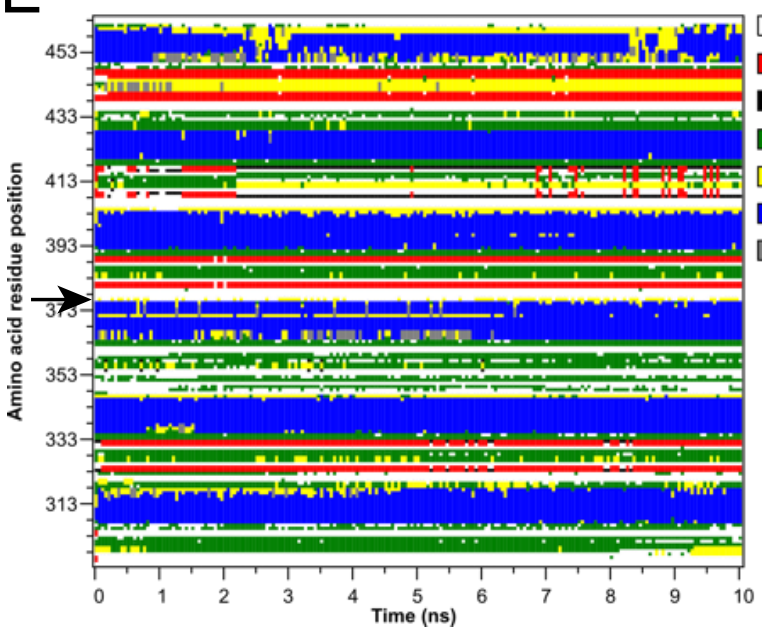

R339Q
B

$\square$ B-Sheet

B-Bridge

$\square$ Bend

$\square$ Turn

A-Helix

$\square$ 3-Helix$$
\text { (1) }
$$

$D$

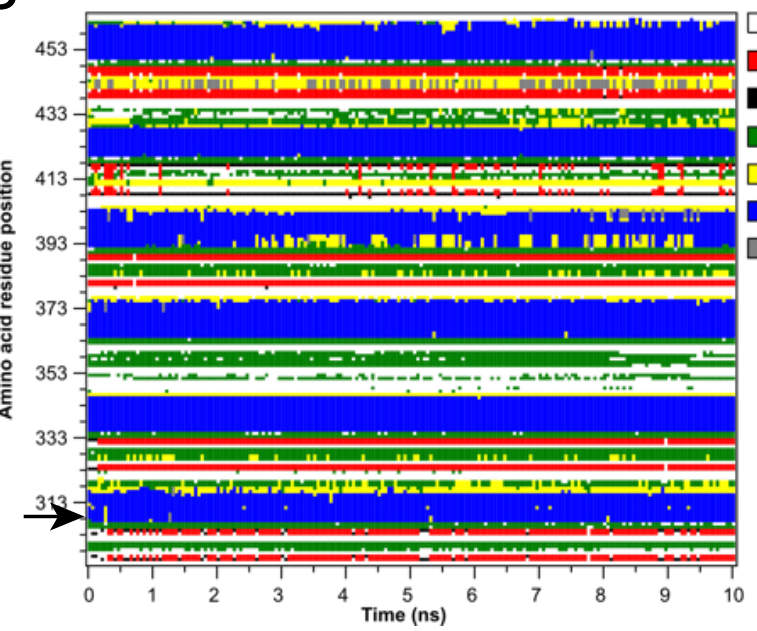

R339W

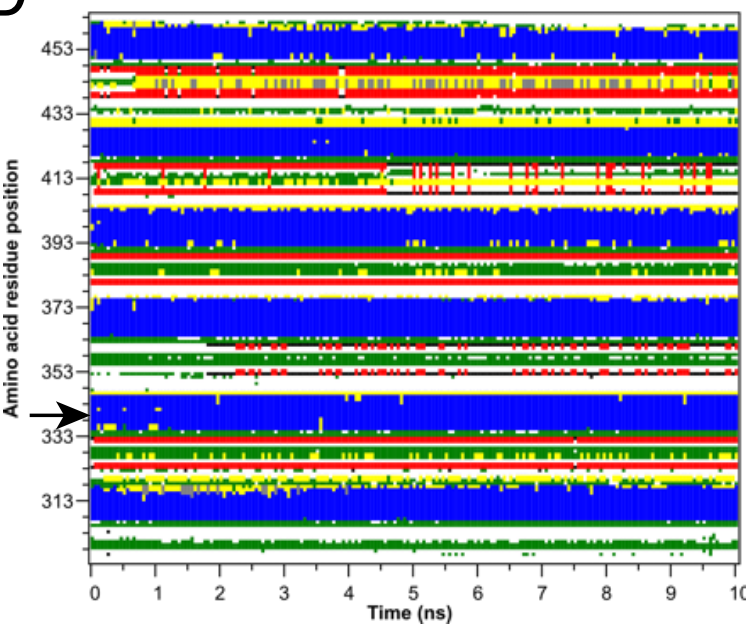

F

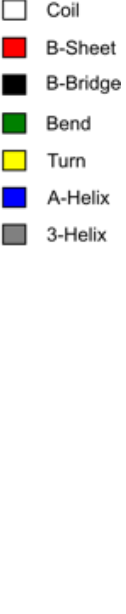

G420D

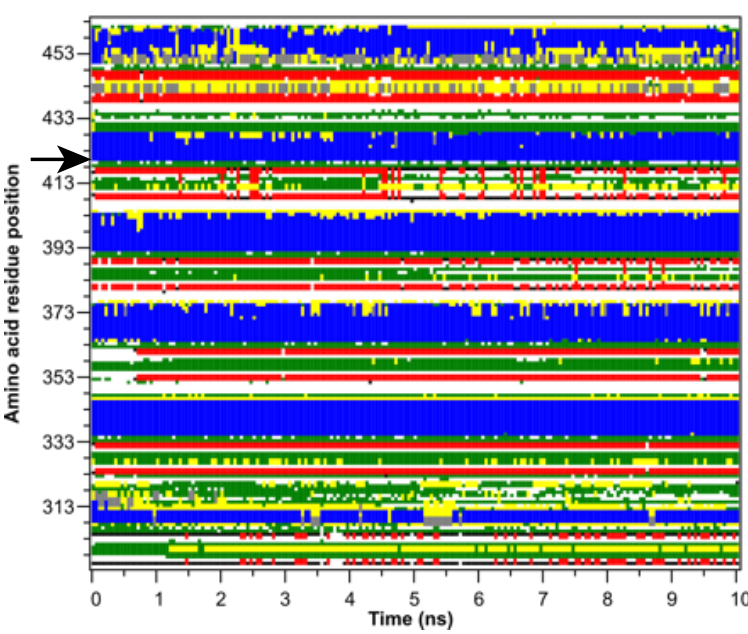




\section{Bailey et al Supplementary Figure 4}
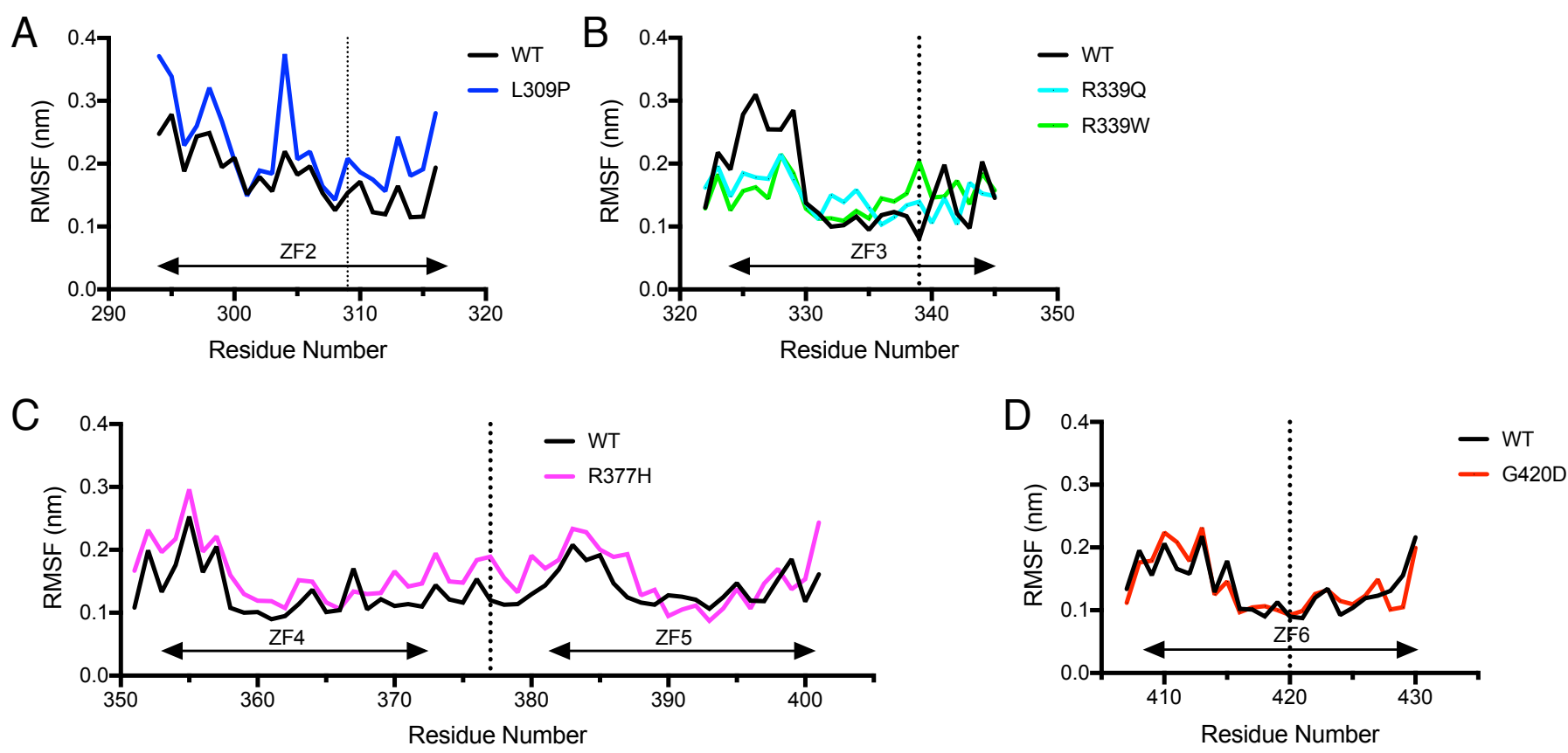